Portland State University

PDXScholar

$11-1-1996$

\title{
Spanish for Health Care Professionals: Language and Culture
}

Mytzi Maryanne Rudolph

Portland State University

Follow this and additional works at: https://pdxscholar.library.pdx.edu/open_access_etds

Part of the Spanish and Portuguese Language and Literature Commons Let us know how access to this document benefits you.

\section{Recommended Citation}

Rudolph, Mytzi Maryanne, "Spanish for Health Care Professionals: Language and Culture" (1996). Dissertations and Theses. Paper 5294.

https://doi.org/10.15760/etd.7167

This Thesis is brought to you for free and open access. It has been accepted for inclusion in Dissertations and Theses by an authorized administrator of PDXScholar. Please contact us if we can make this document more accessible: pdxscholar@pdx.edu. 


\section{THESIS APPROVAL}

The abstract and thesis of Mytzi Maryanne Rudolph for the Master of Arts in Spanish were presented November 1, 1996, and accepted by the thesis committee and the department.

COMMITTEE APPROVALS:
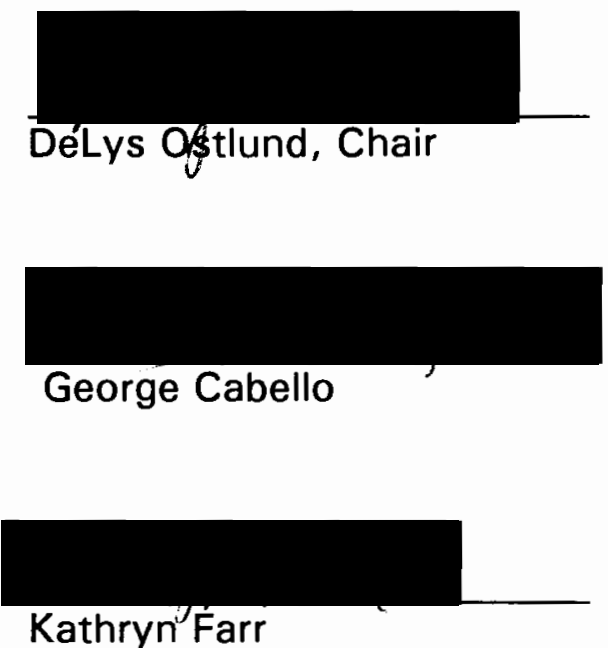

Representative of the Office of Graduate Studies

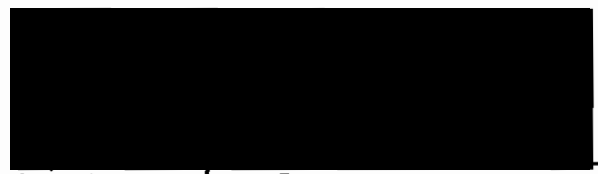

Louis Eltefo, Chair

Department of Foreign Languages

ACCEPTED FOR PORTLAND STATE UNIVERSITY BY THE LIBRARY

by on

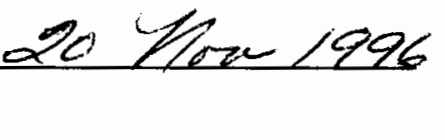




\section{ABSTRACT}

An abstract of the thesis of Mytzi Maryanne Rudolph for the Master of Arts in Spanish presented November 1, 1996.

Title: Spanish for Health Care Professionals: Language and Culture

The purpose of this investigation is to examine formal and informal resources available for teaching Spanish to health care professionals mainly in the Portland, Oregon area.

Seventeen different Spanish-for-health-care-professionals texts are commented on by the author, some of which are the texts used in medical Spanish language classes. The majority of the texts contain little if any instruction on cultural aspects which affect the Latino patient population's health care behaviors and decision making.

With the recent growth in the Latino population there is a greater demand for health care services by Spanish-speaking persons of the Latino community. The author discusses at length current information about the health status of this population, factors affecting access to health care, and language barrier. There is a lack of bicultural and bilingual health care professionals to provide needed health care services to Latinos. One factor is that the percentage of Latino medical and allied health providers is a small fraction of the percentage of Spanish-speaking 
patients in the U.S.

Therefore, Spanish language instruction must be provided to medical personnel who do not have the cultural and language background to provide culturally relevant and efficient health care to Latinos. This language training must incorporate instruction on cultural issues that affect Latino patients' health care. At present very few Spanish-forhealth-care-providers texts and courses have this type of focus.

Exemplary clinical programs specializing in the medical treatment of the Latino population, both inside and outside of the Portland Oregon area, are noted to highlight that effective and culturally relevant medical treatment is possible with adequate training of personnel. Outstanding courses integrating the instruction of both the Spanish language and culture are discussed. Often these courses are not offered as permanent parts of the curriculum. The author gives examples of some of the cultural issues that need to be addressed in language instruction, and makes suggestions for adapting this focus into Medical Spanish instruction. 
SPANISH FOR HEALTH CARE PROFESSIONALS:

LANGUAGE AND CULTURE

by

Mytzi Maryanne Rudolph

A thesis submitted in partial fulfillment of the requirements for the degree of

\section{MASTER OF ARTS}

in

SPANISH

PORTLAND STATE UNIVERSITY

1996 


\section{Dedication}

I dedicate this thesis with love to my parents Ruben \& Eva, and my brothers Bruno \& Ruben Jr.

Acknowledgement

A heartfelt Thank You to my professor and friend DeLys Ostlund for her unwavering encouragement and monumental assistance. I thank Dr. George Cabello and Dr. Kathryn Farr for their careful consideration of my thesis and helpful commentaries. I thank several of my friends for their encouragement and input: Kate Brooks, George Stoumbos, Barbara Roland, Beth Ann Pope, Dr. Cynthia Sloan and Marisela Nyoka. I would like to thank all the professors of the Portland State University Foreign Language Department who have contributed to my education. 


\section{Table of Contents}

Introduction

1. Access to Health Care Services and Language 7

II. Learning to Communicate with the Spanish-Speaking Patient

Population: Formal and Informal Educational Resources

III. Current Tools For Improving Communication Between Patients and Health Care Providers

IV. Cultural Considerations in Patient-Provider Communication 63

$\begin{array}{ll}\text { V. Conclusion } & 113\end{array}$ 
Spanish for Health Care Professionals: Language and Culture Introduction

This project advocates a specialized application of foreign language study. It focuses not only on investigating the development of a Spanish language course specific to the communication needs of health care providers in the clinical settings but also draws particular attention to the necessity of incorporating information about cultural aspects related to the health care of Latino patients.

Understanding and addressing the health care needs of differing segments of patient populations--in this case the Latino patient population--involves interacting with the patients in the language with which they are most comfortable and familiar. The phenomenon under consideration in this study is communication between health care personnel and Latino patients. Such communication contains two principal and recurring factors or constants. The first constant is that the majority of health care providers speak English, not Spanish. These health care professionals may be native English speakers as well as persons who speak English as their second language. Not knowing the Spanish language, they may not be familiar with Latino cultures ${ }^{1}$ as well. Teaching these persons to communicate with Latino patients requires

1 Joseph Hartog defines culture as "the sum total of acquired values, beliefs, practices, laws, customs, traditions, artifacts and knowledge possessed and expressed by a designated group, or 'all human nongenetic, or metabiological, phenomena'" (910-911). 
both Spanish language training and instruction about Latino cultures: a transcultural approach. The second salient factor or constant is that there is an increased demand for use of health services by Latino patients, many of which are non-English speaking.

The difference in languages spoken and unfamiliarity with the Latino culture are manifested as difficulties with communication in the clinical setting which affect the quality and effectiveness of the health care services provided. They are significant factors in health care providers' satisfaction with the quality of care provided to Latino patients and Latino patients' satisfaction with the quality of care that is received. It is well documented in current health care journals that communication barriers and cultural relevancy are factors that affect access to health care services.

Problems in communication between English-speaking health care personnel and Spanish-speaking patients negatively affects health services. In the article "Improving Access to Health Care in Latino Communities" Burciaga et al. clearly state "Poor communications between patients and providers create undue barriers to high-quality care. Few providers speak Spanish, and many lack sufficient cultural competency" (536). When clear communication with patients is not achieved then patients benefit less from the medical services even though more time and effort may be spent due to the language barrier in trying to provide 
adequate services.

The hypothesis in this study is that if a specialized foreign language course can be developed specific to the communication needs of health care providers, then this course will contribute to improving communication, and in turn, improve the effectiveness of health care services provided to Latino patients. The goal of improving the quality of communication between Latino patients and health care providers is something very basic. This has immense ramifications as far as benefits and costs. Better communication between the Latino patients and health care providers would contribute to gains in disease prevention, better health maintenance, earlier medical diagnosis and intervention, all of which affect quality of life and influence health care resource allocation. The deficiency in the aforementioned areas is seen in terms of human suffering and is reflected in soaring monetary costs incurred for the medical treatment of conditions that could have been prevented or treated earlier.

Concern over the health care of the Latino population of the United States has always been relevant but lately it is being addressed as a more timely and pressing issue for two reasons. It has received more attention due to the marked growth in the amount of Latino patients seeking medical treatment. Latino patient population growth parallels the general increase of the Latino population. In addition, because medical care is 
expensive both in the public and private sector, there is sharpened scrutiny over health care services and resource allocation, with interest in keeping medical costs down. In the public sector, more efficient use of tax funds in one area--in health care for example--may mean greater allocation of tax funds for other programs, such as for education or other goverment subsidized institutions. Dissatisfaction with expenditures of money through government programs is fanning the spirit of reexamination and restructuring of services.

As in most businesses, the expenditure of time means either the gain or loss of money. Health care providers who speak Spanish accurately and can provide culturally relevant medical services, save on the amount of time and resources used for exchanging information and providing treatment to monolingual Spanish-speaking or limited-English speaking Latino patients: Resources--such as a medical interpreter--will not have to be used. This, in turn, saves money. The author believes that efficiency in meeting health care needs is linked to foreign language proficiency and cultural competency.

The investigation of pertinent course content as proposed in this study is a step to improve efficiency of services, promote cultural understanding, and help to overcome language barriers. This is in line with one of many concerns addressed in 1992 by the Surgeon General's National Hispanic Health Initiative Commitee meetings held in Washington 
D.C., interested in "developing implementation strategies to overcome the problems of cultural dislocation and assimilation, language barriers. . . " (530).

This study will be divided into four sections. The first reviews information published in current health sciences texts and journals discussing access to health care and illustrating the timeliness of taking a transcultural approach ${ }^{2}$ to health care services, and the role of stress in communication. The second section comments on formal educational resources and published texts for teaching Spanish to health care personnel. The third section discusses current cultural assessment tools used to assess patients and useful to evaluate transcultural curricula. The fourth section discusses various cultural aspects related to the care of Latino patients including folk medical concepts and practices, authority structure, familism, traditional roles, health care professional-patient communication style, fatalism, and locus of control among other topics. These cultural topics are discussed in order for health care personnel to understand the attitudes Latino patients have towards health care and their self-care behaviors (Mardiros 478).

Because language and culture are closely interrelated, language

2 A transcultural approach to Medical-Spanish language instruction promotes cultural sensitivity and plays a part in facilitating culturally congruent health care services. See the definition by Madeleine Leininger on page 34 of this study for a more detailed explanation. 
cannot be studied without keeping culture under consideration. Culture, in turn, has a bearing on the patients' health care behavior. Statistics cited will be both national and regional with areas specific to Oregon and the Portiand metropolitan area.

The intent of this study is to investigate course content for teaching "Medical Spanish" which offers not only a specialized focus for Spanish language instruction but also raises awareness of Latino health care issues and discusses how culture affects different aspects of health care. 


\section{Access to Health Care Services and Language}

Recent health care journals documenting the need to address the health care needs of the Latino population ${ }^{3}$ in the United States advocate a transcultural approach to providing health care services. One facet of this approach calls for instruction in both the Spanish language and culture applicable to the clinical setting. Part of teaching communication is to expand cultural awareness.

For patients who are monolingual Spanish-speakers or who have

3 Some Spanish-speaking persons vehmently disagree with the use of lables such as "Hispanic" or "Latino" to describe the Spanish-speaking population because they are inadequate descriptors which contribute to "racial lumping" (Smart 30) and stereotyping (Andrews 47; Hayes-

Bautista 556). The author acknowledges the use of the label Hispanic in many of the professional publications and has chosen to use this label infrequently due to its innate narrowness of definition. Other labels include "Hispanoamerican, Latin American and Chicano" (Cabello, Thesis Commitee). When referring to the Spanish-speaking target population, an overall term is at times needed. In this study, keeping in mind the limitations of using a label, the target population will be referred to most often as Latino because of its broader focus. Whenever possible, it is best not to use a generic or homogenizing label to describe a population. Richard Root explains this point:

Latinos come from many different countries and each one has a distinct national culture. Whenever possible, the country of origin should be identified and the proper name used in referring to patients, such as Mexican, Salvadorean and so forth. In the United States Latinos share a common language and cultural heritage and, in general, these similarities predominate over national differences. (213) The brief commentary entitled "President's Message: stereotyping in transcultural nursing" by Margaret $M$. Andrews is highly recommended due to its strong advocacy of a transcultural approach to health care services. 
very limited English proficiency, language and cultural differences pose disconcerting and daunting barriers to effective health care. Even though Latinos are the fastest growing ethnic group in the United States, the percentage of Latinos among physicians, nurses and other health professionals remains low.

The 1993 Census reports that there are approximately 258 million people residing in the United States, of which 8.9 percent are Latino (Statistical Abstract 1). That amounts to over 22 million Latino persons here in the U.S. IThe Hispanic Population in the United States: March 199321.

The large Spanish-speaking Latino segment of the U.S. population is not homogenous; rather it is composed of distinct subgroups whose history of residence varies widely. Some Latino families have lived in what is now the U.S. territory for hundreds of years, for many generations. ${ }^{4}$ Other Latino persons have immigrated to this country fairly recently. Patricia Caudel explains:

Hispanics are a diverse group of people of Spanish descent whose ancestors have lived in North America since the

4 To end the Mexican-American War in the Treaty of Guadalupe Hidalgo in 1848, Mexico gave up the territory which now comprises the southwestern states of Arizona, California, Colorado, Nevada, New Mexico, Texas, Utah, and Wyoming (Kirkman-Liff 1403). A large portion of the Spanish-speaking population live in these parts of the country. 
1400 's or who have immigrated more recently from Cuba, Puerto Rico, Mexico, and Central or South America.

Contrary to popular belief, about $70 \%$ of [North] American Hispanics are decendents of peoples who lived here since before the annexation of the southwestern states to the United States in 1848, (40)

Use of the labels "Hispanic" and "Latino" is controversial. "Hispanic" in particular does not accurately describe the Spanish-speaking population which is so rich in diversity. The term "Latino" is also a generic label but is less restrictive. Richard Root notes:

Spain was the dominant colonial power in Latin America, and thus the designation "Hispanics" as a label for Latinos in the United States is not fully acceptable. Hispanic is the official name used by the federal government, but use of this term implies a heritage restricted to Spain. . . . Latino reflects the integration of Spanish, Indigenous and African cultures."

This diversity is reflected in the Spanish-speaking patient population.

The U.S. Census Bureau collects demographic information according to subgroup categorizations, which includes using the label "Other Hispanic." Presumably one of the uses for the demographic data is to help direct the appropriation of public funds for health care. 
However, deficiencies or omissions in data collection--a result of not being attuned to the diversities, both overt and subtle, between the subgroups-have had a negative effect on the allocation of these resources. Caudle observes: "Not recognizing these differences [among the subgroups] has lead to disparities in the allocation of health care resources" (43). A commentary in the Journal of the American Medical Association addresses the issue:

... the large, but inadequately documented, differences among persons of Mexican, Puerto Rican, and Cuban origins in the United States account for poorly focused national policies and limited success in improving the health status of these population groups. (Furino 255)

Population categories are used by the U.S. Census Bureau. 1993 Census data estimated 22.8 million persons of "Hispanic origin" in the U.S. and categorized these persons according subgroups: Mexican, Puerto Rican, Cuban, Central and South American and Other Hispanic. The 1993 Census data by subgroups is as follows:

64.3 percent $( \pm .9)$ Mexican

10.6 percent $( \pm .5)$ Puerto Rican

4.7 percent $( \pm .4)$ Cuban

13.4 percent $( \pm .6)$ Central and South American

7.0 percent $( \pm .5)$ Other Hispanic 
(The Hispanic Population in the United States: March

\section{1}

The information compiled by the Census Bureau provides a rough estimate of the Spanish-speaking subgroups in the U.S. ${ }^{5}$

Demographic statistics alone cannot reflect the diversity among the Latino subgroups. The pioneer transcultural nurse theorist, Madeleine Leininger, admonishes keeping an open mind when it comes to assessing and providing for the health care needs of subgroups: "Health

practitioners should be cautious in making broad generalizations about the health needs of all people . . . and not assume that 'all clients are pretty much alike'" (32).

Health care information often is not specific to subgroups, categorizing Spanish-speaking patients of various origins as Hispanic or

5 The information compiled by the Census Bureau depends upon the technique of self reporting. That is, the person filling out the questionnaire volunteers information about the persons in his or her household. In the 1993 census the Current Population Survey asked persons to report whether they were of the following subgroups: "Mexican-American, Chicano, Mexican, Puerto Rican, Cuban, Central or South American (Spanish countries) [sic], or other Hispanic origin" (Statistical Abstracts 5). Margaret M. Andrews offers an explanation of why these catagories were established:

[T] his government classification was used so that demographic data about traditionally underrepresented populations could be gathered in a systematic manner . . . . Presumably, this was done so decisions about public policies and allocation of resources for these ... population groups could be made in a more equitable way. (47) 
Latino. This makes the information less useful. More accurate health care data would be obtained if the target populations were not homogenized by these non-specific categorizations. The studies specific to subgroups--for instance, the Mexican-American, Colombian, Puerto Rican, Cuban populations etc.--would reveal the commonalities and variations of these populations with regards to health care issues.

The Latino subgroups have drawn more attention partly due to general growth in population. The journal Public Health Reports notes "Hispanics-Latinos [sic] are the nation's second largest and fastest growing minority group" (Treviño 551). The Council on Scientific Affairs predicts that "By [the year] 2000, Hispanics will number an estimated 31 million" (248). Growth in the Latino population concurrently brings an increased demand for health services by members of the Latino community. The health science professions are starting to acknowledge the growing demand for providing health services to this group. The recommendation made by the Hispanic Health Council on Scientific Affairs published in the Journal of the American Medical Association (JAMA) emphasizes "the need for all medical personnel to be knowledgable about Hispanic health care needs" (248). The Surgeon General's National Hispanic/Latino Health Initiative clearly acknowledges the need to improve communication: "Poor communication between patients and providers creates undue barriers to high-quality care. Few providers speak Spanish, 
and many lack sufficient cultural competency" (Valdez 534).

Statistics show that there are few Latino health care workers nation wide: the Portland metropolitan area is no exception. There is great disparity between the limited numbers of Spanish-speaking Latino and/or bicultural health care providers practicing and the huge number of Spanish-speaking patients seeking medical care. National statistics for the first semester of the 1992-1993 academic year show few Latino students in the health sciences. Out of the 257,983 nursing students nationwide, 7,667 or 3 percent are Latino, and of the 66,142 students of Allopathic Medicine nationwide, 3,810 or 5.8 percent are Latino (Health United States 1994 208). As an article in Public Health Reports notes, The need for increased enrollment is evident from the small proportions of selected health professionals who were Hispanics. . . . In 1991, only 4.4 percent of physicians, 2.4 percent of registered nurses, 2.7 percent of dentists, and 3.2 percent of pharmacists were Hispanics. (Treviño 555)

The fact is that in the United States there are relatively few Latino health care professionals in comparison with the number of Latino patients receiving medical treatment. To attempt to analyze why so few Latinos seek careers in the health sciences is an issue involving complex social, economic, and cultural factors and will only be addressed briefly. Public Health Reports offers a succinct commentary: 
Hispanics-Latinos [sic] in the United States as a group have not achieved high levels of formal education because of multiple sociocultural barriers. Hispanics-Latinos continue to enter elementary school later, leave school earlier, and receive proportionally fewer high school diplomas and college degrees than do other Americans. . . . As of 1991, only 51.3 percent of Hispanics-Latinos ages 25 and older had completed 4 years of high school or more, compared with 80.5 percent of non-Hispanics. (551)

Enrollment of Latinos in the higher educational system is far more limited than for non-Latinos. This affects recruitment of Latino students into the health professions in the United States.

The 1990 Census Equal Employment Opportunity (EEO) detailed report for the Portland, Oregon area documents that of the 3,922 physicians in Portland, only fourty-five identify themselves as Hispanic. The report shows while there are a few Hispanic health care professionals--fifteen health diagnosing practitioners, twelve pharmacists, seven dietitians, three respiratory therapists and twenty-one physical therapists--there are no Latino dentists, optometrists, podiatrists, occupational therapists, speech therapists or physician assistants.

According to this same report, of the 11,472 registered nurses in Portland, 177 identify themselves as being Hispanic. The Oregon State 
Board of Nursing offers somewhat differing statistics. In 1993 there were a total of 31,045 registered nurses licensed in the State of Oregon, 220 of which identified themselves as "Spanish" at the time of applying or reapplying for licensure. The licensure application for registered nurses requests demographic information and provides several descriptors from which to answer. The demographic categorization "Spanish" does not distinguish between nurses who speak Spanish and those who do not. When examining these statistics one must keep in mind that not all health care providers who identify themselves as "Spanish" speak the Spanish language. There are also a few health care providers who speak Spanish who are not Latino. It also cannot be assumed that bilingual Latino health care providers automatically take special interest in providing services to persons of the Spanish-speaking community.

The aforementioned data support the observation that most of the medical personnel in the U.S. treating Latino patients are non-Latino. Michele Eliason comments: "Since the health care system is primarily (and historically) made up of white people from the middle-class, the health care system and the dominant culture are interrelated and overlapping" (226). Dahlia Rojas makes a similar observation: "Over 90 percent of the nursing workforce are non-Hispanic European-American women from working-or middle-class backgrounds" (258). The majority of health care personnel is English speaking and of different cultural 
background from the Latino patients to whom they treat. ${ }^{6}$

There is a wide language gap between the Latino non-English or limited-English speaking patient population and the English-speaking health care providers of Anglo-European background. Woloshin explains: "The U.S. Department of Health and Human Services [DHHS] office for Civil Rights uses the term 'limited English proficient' to define the portion of the population that is non-English speaking or limited-English speaking" (724). The language gap affects the quality of the interaction between English-speaking medical personnel and Spanish-speaking patients seeking medical attention. It affects the ability of health care providers to obtain needed health assessment data from the Spanish-speaking patients and relay health care information to the Spanish-speaking patient unless there is an interpreter present. ${ }^{7}$ Monolingual English-speaking medical personnel who are knowledgable and qualified to instruct the patient population cannot communicate due to the language barrier. In the best of scenarios, communication is facilitated through the use of professional medical interpreters. However, making arrangements for the use of these

6 The citation by Eliason brings to attention ethnicity and class. Gender is also an issue in the quotation by Rojas because the majority of persons in the Nursing profession are women.

7 Several sources recommend the AT\&T Language Line (800-752$6096)$ which provides medical interpretation services by telephone as an option (Rankin 827; Woloshin 726; Putsch 51). 
professionals requires extra time, planning and resources. Health care institutions are required by federal law to provide interpretation services:

The DHHS Office for Civil Rights views inadequate interpretation as a form of discrimination. This view has its origin in the Civil Rights act of 1964. . . The office also requires DHHS-funded health programs to provide patients with limited English skills access to services equal to those provided to English speakers. Programs that do not comply risk loss of all federal funds, including Medicare and Medicaid payments. (Woloshin 725)

The institutions providing medical care, then, have the legal obligation to provide quality care to non-English speaking patients, part of which includes the provision of adequate language services. As described by $\mathrm{Dr}$. Robert W. Putsch, "Title VI of the Civil Rights Act of 1964, as well as Section 504 of the Rehabilition Act of 1973" were instrumental in the development of language services for patients. Dr. Putsch also points out that in 1979 Medicare passed a policy which gave language services a financial backing. The policy states: "The costs incurred for bilingual services are allowable provider costs" (3345). As Dr. Putsch explains, "These policies allow institutions to incorporate interpreter services into their fixed operational costs" (3345). The burden of arranging for and financing adequate interpretation services is upon the medical institution 
and not the individual patient: "Interpretation costs cannot be directly passed on to monolingual or hearing-impaired individuals" (Putsch 3344). Despite the legal and financial provisions that exist for the development and use of professional interpretation services, oftentimes informal interpretation resources are used by patients. It is common that patients' bilingual family members or friends are present and offer to assist with interpreting for the patient. This is one form of what is called ad hoc interpretation (Woloshin 724).

To the health care provider the ad hoc interpreter's presence and willingness to assist oftentimes seems a convenient resource to use; however, this practice is to be discouraged. There are numerous problems associated with the use of untrained non-professional interpreters, such as unreliable accuracy of interpretation and problems with confidentiality. Dr. Putsch also mentions problems with "bad paraphrasing and frank omissions [of information]. . . . [E]rrors most frequently involve names, omissions, additions, substitution of terms, incorrect numbers (dates, quantities, etc.) and garbling of the message" (3345). These are only a few of the problems and issues with regard to translation as documented in the health care literature. The utilization of professional medical interpreters is effective, but is not as efficient as receiving information first hand from a bilingual, bicultural health care provider. This underscores the need for a transcultural focus in health 
care education which encourages the development of foreign language ability and cultural competence.

Health sciences professionals studying Spanish play a vital part in recognizing the uniqueness and commonalities between subgroups and making medical care culturally congruent for Latino patients. Subgroupspecific health research can help to make medical treatment more effective by guiding the development of culturally congruent treatment interventions. It can also be influential in improving the allocation of health care resources to this target patient population because these data can be used to guide the funding and instigation of culturally-sensitive public health programs.

In the professional health sciences journals several articles note the limited availability of current health status data on the Latino population in the United States. In fact Marin et al. sharply point out that there is a "Dearth of research instrumentation" and continue on to say:

A large number of research projects that include Latinos are currently being carried out without adequate attention to the culture of the respondents; their group-specific attitudes, perceptions, expectations, norms and values; or the requirements of appropriate linguistic adaptation. Overall, the lack of appropriate research instruments limits the availability of valid and reliable data and deters the 
development of further research. (547)

The collection of information that is "without adequate attention to the culture of the respondents", is one of the many factors that lead to inadequacies and miscalculations in data collection.

Due to the fact that differences according to Latino subgroups is not well accounted for in health studies data, this limits the information available about the overall state of health for the population. The article "Hispanic Health in the United States" published in the JAMA gives the following overview of the numerous illnesses that affect the Latino population. Illnesses that are prevalent include heart disease, cancer, stroke, gallstones, alcoholism and cirrhosis. Compared to the general U.S population, Latinos in the U.S. have increased incidence or occurence of stomach cancer, esophageal cancer, gallbladder cancer, cervical cancer, death due to narcotic addiction, violent deaths due to homicide, teenaged pregnancies and lack of prenatal care. Illnesses for which there is increased risk or propensity are diabetes of greater severity, diabetic retinopathy, obesity, higher levels of cholesterol and triglycerides, undiagnosed hypertension, untreated hypertension, lung cancer, tuberculosis and human immunodeficiency virus infection (248, 250-51). The article "Health Status Among Hispanics: Major Themes and New Priorities" also published in the JAMA adds to the extensive list above. It mentions the prevalence of diabetes type II and higher incidence of end 
stage renal disease, preventable childhood diseases, bacterial gastrointestinal diseases, depression, dental caries, periodontitis and communicable diseases. It reports higher risk for breast cancer in women. It also speaks of the existence of substance abuse, acquired immunodeficiency syndrome and injuries due to violence (256).

Latinos represent a diverse patient population with regard to medical diagnoses. Studies show that for various reasons segments of the Latino population underutilize health care services including preventive health care services such as routine physical examinations, screening, and immunizations. Often people wait to seek medical treatment and enter the health care system once illnesses are well advanced.

The chronically underserved population requires more intensive medical care when care is finally sought by them, which adds to the overall cost of medical services. Attention to preventative care and health maintenance by bilingual and bicultural personnel would better serve the patient population and, in the long run, decrease the cost of medical care to this population.

It is recommended that outreach programs teach the promotion of health and the prevention of disease. This strategy of providing health education is in line with the aforementioned goal of keeping health service costs down by encouraging illness prevention. Education about health maintenance and early treatment intervention would help decrease the 
occurrence of acute illness and the treatment costs therewith incurred.

Studies investigating the delays in seeking medical care reveal economics as a major factor in this decision, given that many Latino patients do not have health insurance. Linda Wray finds that "socioeconomic status is related to health care use through access--that is, in financial capacity and private health insurance coverage" (358). Patricia Caudle points out that "lack of financial resources represents the most important deterrent to the use of health care services" (43). The Council on Scientific Affairs reports: "Low incomes and lack of health insurance restrict Hispanic access to primary health care more than any other variables" (250). Valdez points out the consequence of delaying medical treatment due to fear of out-of-pocket expense:

Uninsured Latinos experience high rates of hospitalizations for conditions (for example, asthma, diabetes) that could have been prevented, treated, or controlled with inexpensive and timely primary care. (535)

Burciaga Valdez offers an estimate of the number of uninsured Latinos in the U.S.:

[M]ore than 7 million Latinos younger than 65 years [old] are completely uninsured. ... In the last 10 years, the number of uninsured Latinos increased by 151 percent, whereas Anglo numbers increased by 32 percent. 
(Valdez 534-536)

Many low income Hispanics without health insurance are employed in low paying temporary or part-time jobs that do not offer insurance for medical care as an employment benefit (Hispanic Health 249; Caudle 43). Julia Solis describes further:

The "working poor" represent a segment of the Hispanic population that is especially vulnerable to access barriers in that they are neither able to afford the rising cost of medical services nor eligible for assistance through governmental programs. (11)

The 1990 Surgeon General's National Hispanic/Latino Health Initiative examined this problem of national concern, pointing out the paradox in the situation:
Although we [the Latino population] are the most highly employed minority, more than one-third of us have no health insurance. . . Twenty-five percent of Hispanic-Latino families live in poverty, with a median family income of $\$ 23,431$ as opposed to $\$ 36,334$ for non-Hispanics-Latinos [sic]. (529)

This dilemma is not exclusive to the Spanish-speaking population. ${ }^{8}$

8 Burciaga Valdez estimates that "nationally 35 to 37 million Americans do not have health insurance ... . "(534). 
Some low income Latinos are eligible to use the government-funded Medicaid program. Latinos at the working-poor income level who do not qualify for Medicaid have three options: "pay high prices for medical care or receive charity care or no care when it is needed" (Caudle 43).

Medicaid is a useful but expensive program. ${ }^{9}$ It is estimated that nationwide in 1992, 5.0 million Hispanics had Medicaid coverage; 10.1 millon had Private Health Insurance; 1.5 million had Medicare; 7.4 million had no health insurance (Statistical Abstract 118). In Oregon there is the Oregon Health Plan, which has strict eligibility restrictions. Persons in the working-poor income bracket generally do not qualify for this assistance. Of the people residing in Oregon there were a total of 295,000 Medicaid recipients in 1992 (Statistical Abstract 115). One can speculate that the number of Hispanics in Oregon receiving welfare reflects the number receiving Medicaid since "medicaid eligibility is automatic for almost all cash welfare recipients" (Statistical Abstract 108). Due to the quantity of money spent on Medicaid, it is in the public interest--both of the tax payer and medical service recipient--for services to be rendered in a more efficacious and culturally congruent manner. The development of bilingual and bicultural skills in health care professionals who work with Latino

9 In 1993 the nationwide cost for all persons enrolled in Medicaid, Latino and Non-Latino, "totaled $\$ 102$ billion for 33.4 million recipients" (Health United States 1994 8). 
patients is an obvious major step to this end.

It is appropriate, at this point, to delve further into the topic of patients' language ability--both reading and writing skills in the English language and in their native language Spanish--because often it has bearing on patient's socioeconomic situation, which affects accessibility to health care services. Educational background is a socioeconomic factor. Among the 22 million Latinos in the U.S., there is a wide range of educational levels.

The extensiveness of the education received prior to coming to this country is related to the financial situation. There are persons who have recently immigrated from countries whose educational system required the study of a second language. This is especially true if they had the financial resources to attend private schools. These persons may have chosen to study the English language as their second language and use the language at various levels of proficiency.

The writer makes the assumption that Hispanics with a greater level of education are more likely to be bilingual. This is a key point. Due to their more extensive educational background they may attain a higher income level. Persons of higher income--that is to say, people with health insurance and who can afford out-of-pocket expenses for medical services--have better access to health care.

Bilingual persons of higher income do not face the degree of 
difficulties caused by language barriers that monolingual Spanish-speaking persons have to confront. Bilingual latinos are most likely to be bicultural. They also may be more acculturated to the dominant U.S. culture, readily adopting it instead of maintaining the cultural traditions from their country of origin. In time and through numerous types of interactions in society, bilingual speakers have internalized the ways of life, values and norms which stem from the dominant culture, mainly non-latino European influences such as "Western American values of individualism, selfreliance, self-sufficiency, independence, autonomy, self-control, selfmanagement, self-directedness, and related self-modalities" (Self Care 2). In a dynamic process these values and ways of being have helped to shape the behavior and attitudes of subsequent generations, creating a common thread of shared culture found among persons residing in this country.

Spanish-speaking patients of low income who are new to this country or who are not well acclimated to the dominant culture face many more problems in arranging for health care services. Monolingual Spanishspeaking patients of high income in the U.S. face a language barrier but do not have economic difficulties paying for the needed services.

There is the case in which recent immigrants are in the stage of just starting to learn the language. There are other immigrants who have not learned English because they live in a socio-cultural environment in this 
country in which they are able to manage sufficiently without having to speak English. Not being bilingual puts these people at a disadvantage when obtaining medical services because the majority of the personnel in the health care system are monolingual English-speaking, and services are more readily available to persons who speak English.

The author makes the assumption that persons of the Latino population who are of lower income have less of an educational background and are less likely to be fluently bilingual. This makes bilingual health care providers all the more valuable to this patient population.

Because there are so many Spanish-speaking people of low income who enter the health services system, a portion of this study will address some of their health-care-related concerns in particular. One problem in health data collection and treatment of persons of low income is the limited accessibility to this percentage of the Latino population, for example persons living in areas of urban poverty, migrant workers, persons who live in the colonias ${ }^{10}$ or "unincorporated settlements"

${ }^{10}$ The colonia communities "often lack septic tanks, sewers, or running water, and outdoor privies commonly abut water wells, making most of the water unfit for consumption" (Warner 242). An article published in 1991 by David C. Warner notes: "It was estimated in 1988 that more than 110,000 persons lived in up to 600 of these colonias in Texas" (242). A more recent article by Martha E. Burk et al. published in 1995 notes the following:

Colonias are unincorporated, rural subdivisions that offer 
(Warner 242).

A large portion of the Latino population that is medically underserved is the migrant worker population. Therese Martaus' investigation of the overall migrant worker population, using estimates from the DHHS, found that there are "at least 800,000 migrant workers in the United States, more than $75 \%$ of whom are Mexican or Mexican American" (32). Ketty Mobed quotes an estimate from Legal Services Corportation in Washington D.C. stating that " 5 million migrant and seasonal agricultural workers live and work in the U.S." (367). Thus, there is a great discrepancy in the estimates of how many persons work in seasonal and migrant labor and who consequently are at risk for certain illnesses due to known common working and living conditions. Mobed makes the point that due to the

transient nature of the population, their migration into and out of the U.S. [there is] undercounting of those workers who meet the legal definition of a migrant but who do not fit

substandard sanitary and living conditions to mostly Mexican-American indigent families. It is estimated that over 140,000 persons live in 500 to 600 colonias in Texas, with roughly $80 \%$ in the lower Rio Grande Valley and about $20 \%$ in Webb (Laredo) and El Paso counties. Since 1985, the total number has increased, . . . such settlements are common and have expanded rapidly to accommodate the growing population. (47) 
ethnic and demographic stereotypes or occupational classifications, and the desire of many immigrant workers to avoid contact with government agencies. (367)

To the extensive list of common health problems found among the Latino population in general can be added the occupational health problems of agricultural workers. These include the following: accidents, pesticide-related illnesses, musculoskeletal and soft tissue disorders, dermatitis, noninfectious respiratory conditions, reproductive health problems, health problems of children of farm workers, climatecaused illnesses, eye and ear problems (Mobed 367).

Oregon is affected by the social and economic challenge of providing health care services to a large group of low income Latino people, many of whom are seasonal or migrant workers who are vital to sustaining the agricultural industry of the state. A consultation with regional economist, Gary Sincick, at the Oregon Employment Department in Portland, Oregon reveals an estimate that 31,413 seasonal agricultural workers were employed in Oregon in June 1995, considered a peak time for this type of employment. This number is just a fraction of the migrant worker population in the U.S.

This is a factor in medical conditions being untreated for prolonged periods, minimal use of preventative and health maintenance care, all of which contribute to public health illnesses--such as tuberculosis--that are 
endemic to certain subgroups and medical care for illnesses that are far advanced and thus more costly to treat.

The professional challenge--based on medical ethics as well as a the consideration of cost control--is to provide effective health care services to the large segment of the Latino patient population that faces difficulties due to language barrier and insufficient means to pay for health care. Recently on the West Coast concern and dissatisfaction over the cost of providing government-funded social services, including Medicaid, has turned into a politically-charged topic. The controversy has been over California House Bill 187 and Oregon House Bill 2933. Both House Bills mandate a change in eligibility for Medicaid and other social services by requiring the verification of immigration status. Modification of current eligibility requirements would affect many Latinos receiving government assistance. Public policies regarding health care costs are at the center of heated debate, and the issues will continue to affect Latinos as long as a certain segment of this population cannot afford health care services. Linda Wray brings this issue to the forefront, stating that "efficiency and costs remain priorities in the 1990's, consumers, health care professionals, and policymakers are increasingly emphasizing quality-ofcare issue, including access for all" (359).

There appears to be a discrepancy between the desire to decrease the overall cost of health care expenditures, especially government-funded 
services, and the need to provide basic medical care. However, what seems contradictory--providing basic medical treatment to the uninsured-in fact would help keep the overall cost of health care services down. Many studies recommend improving accessibility to out-patient clinic services and establishing health education programs targeting the Latino community. Innovative out-patient clinic services and educational programs providing culturally sensitive services have been documented in the health sciences literature. One such program, Project Vida in Texas, provides services to 10,000 documented and undocumented Mexican immigrants and has been proven to be very effective (Skolnick 1445-6). Another successful outreach and education program is Un Comienzo Sano in Arizona which provides pre- and post-maternity services in order to promote "better birth outcomes" (Meister 38). Another noteworthy specialty clinic providing fairly comprehensive services in Texas is $\underline{S u}$ Clinica Familiar. ${ }^{11}$ These specialties include adult medicine, pediatrics,

\section{Familiar:}

${ }^{11}$ Burk gives details of the services available through Su Clinica

A full scope of primary care services is provided to improve the health status of mostly indigent residents. . . Of the 30,000 clients using SCF services annually, $94 \%$ are Mexican American, and approximately $50 \%$ are uninsured. . . 'One-stop shopping' is offered through various in-house support services, including case management, outreach, integrated eligibility, social services, nutrition counseling, pharmacy, laboratory, radiography, and referrals. (45) 
obstetric, gynecologic, and dental services (Burk 45). In Oregon, there exist a few clinics that specialize in providing primary care to Latino patients: La Salud clinic in Woodburn, The Virginia Garcia Clinic in Hillsboro, and La Familia Sana clinic in Hood River. Many of the low income population treated at these clinics are seasonal agricultural workers. For this type of outreach programs to exist there needs to be health care providers with the linguistic and cultural competency to provide the necessary health care services and education. Linguistic and cultural competence is not only important in the outreach educational settings but is just as significant in the clinic and hospital settings.

Language differences present difficulties not only during medical treatment but also before receiving medical attention, during the process of trying to arrange for treatment. One of the findings from the nationwide Hispanic Health and Nutrition Examination Survey (HHANES) was that language is a significant factor in obtaining medical services. Julia Solis notes from the HHANES that the ability to speak English affects patients' access to health care services, calling it an "important predictor of utilization" (16).

The HHANES findings document that the language barrier places monolingual Latino patients at a disadvantage. Their access and utilization of health care services is reduced by their inability to speak English. Several other studies describe language differences in the clinical 
setting as a barrier to accessing health care services (Caudle 43; Health Status 256; Surgeon General's 530; Thorngren 39). As Steven Woolsin states, "Language can be a formidable barrier to accessing and receiving health care" (724). This is significant because due to the language barrier and possible miscommunications, prescribed treatments are less effective, if not altogether ineffective. Instead of helping to resolve the medical problem, the resources expended may contribute to illnesses becoming worse, which is a waste of time, effort, and resources. The results of miscommunication are frustrating to the patients and costly. Unresolved medical problems necessitate the repeated use of medical care services. Every time the above-mentioned cycle is repeated, the cumulative cost of treating the patient's condition increases. Ineffectively managed conditions can develop into acute or chronic illnesses. Interaction with Spanish-speaking Hispanic patients would be much more effective if the patients were spoken to in the language with which they are most comfortable and familiar. In order to achieve this, medical personnel need to be taught Spanish as appropriate to the clinical setting. ${ }^{12}$

Expanding the student's cultural awareness involves exposure to other perspectives, beliefs, other ways of doing things, and ways of

12 Health care providers who deal predominantly with non-English speaking people from other cultures--e.g. Russian, South East Asian etc.-also need multilingual and multicultural training to better understand and serve the patient populations they treat. 
living. In other words, instruction in language and culture can help students develop a sense of "ethnorelativity," a term coined by Michele Eliason which she defines as "the ability to conceive of alternative viewpoints and to respect the beliefs of another culture even though they are different from one's own" (226). Expanding cultural awareness is related to the principles of a transcultural approach, which encourages the acknowledgement of and respect for cultural variation and looks to incorporate it into treatment plans. According to $M$. Leininger, the leading transcultural nurse theorist in the U.S., the transcultural method consists of:

[The] comparative study and analysis of different cultures and subcultures with respect to nursing, health, and illness caring practices, beliefs and values, with the goal . . . to provide culture-specific and culture-universal nursing care practices. (Essential Goal 12)

Awareness of differing cultural practices is helpful in formulating culturally congruent approaches to patient care.

A culturally congruent approach increases the probability that the patients will follow through with the treatment regimens as prescribed, making the health care service more effective. Culturally sensitive practices have immediate and long-term positive ramifications. One immediate benefit from interactions with culturally aware Spanish- 
speaking health care providers is that such interactions help to decrease the patient's stress associated with contact with the health care system and the illness.

IIIness is a stressful event. It is a disruptive stressor. Stressors are defined as "the agents placing demands upon the body which can precipitate stress" (Luckmann 32). Cecil Helman describes the biomedical concept of stress:

Not all stress is harmful to the organism: at a moderate level (eustress) it has a protective and adaptive function. At a higher level (dystress), though, the stress response can cause pathological changes, even death. The actual environmental influence--whether physical, psychological or sociocultural--that produces stress, is termed stressor. (249) The effects of stress, then, can range from helpful to harmful. A certain amount of stress can increase alertness, perception and improve attention. This level of stress is positive for the patient in her or his efforts to communicate. Dystress, on the other hand, brings on physiological effects that negatively affect patient's communication. IIInesses range from mild inconveniences to serious crises and can affect persons on an emotional level. The combination of physiological and emotional stress may cause anxiety which, whether moderate or severe, affects cognitive functioning. The body's systemic reaction to 
stress is described in the stages of the General Adaptation Syndrome (GAS) or Stress Syndrome, involving the nervous and endocrine systems (Lewis 1145; Luckmann 33). This GAS "involves a wide range of physiological changes" (Helman 249). When the patient comes into contact with the health care system, his or her body is affected by the symptoms of GAS in addition to the symptoms of the primary illness for which the patient is seeking medical attention.

Louise Shives lists some of the "more common physiologic symptoms" of stress:

Elevated pulse and blood pressure, dyspnea or hyperventilation, diaphoresis, vertigo or light-headedness, blurred vision, anorexia, nausea and vomiting, frequency of urination, headache, insomnia or sleep disturbance, weakness or muscle tension, tightness in the chest, sweaty palms. (237)

Another type of reaction to a stressor is psychological. As Helman notes, [there are] "psychological responses or coping strategies of the individual confronted by a stressor. These range from an initial alarm and shock state with feelings of anxiety or of being threatened, to attempts to cope" (250). Emotional stress can be precipitated by physical discomforts of the illness along with the inconveniences and disruptions the illness causes in the patient's daily routine and ability to fulfill role obligations. It 
can also be caused by life changes due to developmental or situational events other than illness.

Latino patients, if they are limited-English or monolingual Spanishspeaking, experience stress on various levels when they come into contact with health care professionals who do not speak Spanish. One of the stressors is that contact with mostly English-speaking health care systems can be perceived as threatening by the patients and thus be anxiety-provoking. A patient may not only have difficulties with language and cultural barriers--as the English language and culture may yet be foreign to them--but in addition, the health care setting itself may cause them a certain degree of "culture shock" because of the unique nature of the environment. Cumulatively this only adds to the stress of illness and the disruptions that it causes in patients' lives. Mary Thorngren speaks of "Hispanics' mistrust of the health care system born of repeated negative experiences" (40). Indeed, even to many English-speaking patients the clinic and hospital settings cause feelings of discomfort and anxiety.

The level of anxiety experienced can deplete the Latino patient's capacity to use the limited English that he or she knows and adds to the physiologic complexities of the illness. Bilingual and bicultural persons experience less stress when faced with situations in which there may be "cultural conflict" as they are better able to adapt (Leininger, Becoming Aware 33). Shives defines anxiety as "feelings of uncertainty, 
uneasiness, apprehension or tension that a person experiences in response to an unknown object or situation" (588). Margo Creighton, in turn, defines it as an "uncomfortable feeling or tension which can be vague and/or intense. It occurs as a reaction to some unconscious threat that the person is experiencing" (17). The uncertainty of the illness and course of treatment, the unfamiliarity with the beauracracy, rules, norms and procedures of the clinic or hospital environment, language barrier, as well as other factors all can provoke anxiety in patients.

A person's anxiety level can even escalate to such a point that the ability to use one's own native language is impaired. Shives lists symptomatology that occurs with varing levels of anxiety:

Level 2: MODERATE ANXIETY. During this stage narrowing of the ability to perceive occurs. The person is able to focus or concentrate on only one specific thing. Level 3: SEVERE ANXIETY. The ability to perceive is reduced, and the focus is on small or scattered details. Inappropriate verbalization, or the inability to communicate clearly, occurs at this time. ... [There are] decreased intellectual thought processes. . . . [T]he person experiences feelings of purposeless. (238)

Level 4: ANXIETY, PANIC STATE. There is complete disruption of the ability to perceive. Disintegration of the 
personality occurs as the individual becomes immobilized, experiences difficulty verbalizing and the inability to focus on reality. (238)

Creighton similarly outlines symptomatology of anxiety states in relation to communication:

Moderate anxiety: selective inattention; a decreased ability to communicate or perceive the environment unless it is pointed out; learning can still occur but at this level it must be directed.

Severe anxiety: a drastically reduced ability to perceive and communicate details; the whole is perceived, ... the connection between details cannot be made; learning cannot occur at this level.

Panic: any detail which is perceived is elaborated and distorted; the person is unable to communicate or function; learning cannot take place. (17)

From the above it can be seen that anxiety affects language use. If anxiety can make communication in one's native language difficult, it can certainly affect a person's ability to use a second language, exacerbating problems with the language barrier.

Bilingual patient's communicative abilities are affected by stress as well. During a moderately stressful situation, a patient who is fairly 
proficient in both languages may find that he or she can think and communicate much clearer in his or her native language than in the nonnative language. Linda Haffner makes an interesting comment with regard to this:

Family members--and patients, for that matter--similarly untrained and inexperienced [in interpretation] may suffer a dramatic decline in English proficiency when confronted with the stresses inherent in the context of illness. It has been observed that the "language of our childhood remains the language used during times of intimacy and stress." (259) In a clinical situation where a Latino patient of limited English proficiency is experiencing physiological and emotional stress, it is most likely that he or she may hear and understand only fragments of what is said, may be forgetful, may have increased difficulty using the English language, and/or may feel overwhelmed.

Moderate or severe anxiety can affect a limited English-speaking patient's cognition and ability to communicate. Anxiety may cause a setback in the patient's capacity to comprehend and speak English. The result of this setback is that the patient may be more easily pushed beyond his or her English language capacity, experiencing frustration and linguistic breakdown. The increased difficulty in speaking English under stress can contribute to the patient's feelings of being overwhelmed in the 
health care setting. A coping strategy for the patient is to resort to using or prefer communicating in Spanish, their first language (Orque et al.

123). During an episode of high stress, the patient may appear to have less of an overall English ability than would be present under less anxietyprovoking circumstances. The patient may recognize his or her temporary lapse in ability to speak English and feel frustrated about this. One factor builds on another, escalating the discomfort of the situation.

Bilingual and bicultural health care personnel play a significant role in helping patients to overcome the language barrier. One way of helping is to create a more therapeutic environment: either through use of medical interpreters or the facilitation of medical professionals' learning of Spanish and sensitivity to health care issues of the Latino population. Bicultural knowledge improves communication and assessment skills by helping one understand the patient's point of view. What usually is thought of as communication with a Latino patient--the relaying of biomedical information or treatment instructions in Spanish usually done through an interpreter--is a necessary part of working with Latino patients but it is not enough. The interaction and communication process is placed on a higher, more effective level when the health care personnel providing the primary care to the patient can speak Spanish and consider the implications of culture on the patient's health care practices. Burk speaks of this saying, "use of a client's primary, or preferred, 
language is of utmost importance to enable the client to gain access to care, process health information, and adhere to prescribed regimens" (40). Aaron Manson conducted a study with regard to "language concordance" and compliance of the Latino patients with prescribed medical treatment. He concludes:

Patients with bilingual physicians ... were more likely to take their medication and keep their appointments with their primary care physician. . . . [T]he inability of physicians and patients to speak the same language wastes ambulatory care resources and may be related to use of more intensive, costlier hospital services. $(1127,1128)$

These study results supported the idea that speaking the patient's language is a factor that helps to effect positive treatment and follow up behavior by patients.

A long term positive ramification is that the cost of health care services is reduced. An immediate benefit is that it mitigates the stress that is caused by contact with the health care system and illness, making the illness situation more managable for the patient. A therapeutic environment helps to reduce the patient's anxiety level, thus helping to improve the patient's coping ability and opportunity for healing. 
II. Learning to Communicate with the Spanish-Speaking Patient

Population: Formal and Informal Educational Resources

As the medical community comes to recognize the problems caused by poor transcultural communication, more resources are being dedicated to ameliorate cross cultural medical services. There are formal and informal resources available for learning about cross-cultural or transcultural patient care principles. Some outstanding examples of formal resources documented in the health care journals include programs at various universities in the U.S. The University of Connecticut School of Nursing in 1995 published an article entitled "A Model for Delivering Culture-Relevant Care in the Community" and established a pilot program ${ }^{13}$ "designed to infuse Spanish into the curricula of the Schools of Nursing and Allied Health at the University of Connecticut" (Wright, Q\&A Spanish 190). The University of Miami School of Nursing published an article in 1991 about incorporating a transcultural framework into their undergraduate and graduate level nursing curricula and offered a faculty development program whose objective was to "increase faculty skills for

${ }^{13}$ Barbara D. Wright Associate Professor at the University of Connecticut was kind enough to send the author information about the pilot summer program "Spanish 190: Language, Culture, and Health in Spanish-Speaking Societies." This program so far has been held for two sessions: July 24 to August 11, 1995 and June 3 to June 21, 1996 at the Storrs campus. The author was invited by Dr. Wright to be an outside observer and to help evaluate the program. Unfortunately the author could not attend either of the sessions. 
integrating the concepts of culture into clinical practice settings"

(DeSantis 301). Rush Medical College in Chicago in 1994 published an article about the "Spanish Language and Hispanic Cultural Competence Project" that was offered to medical students as a clinical elective. These programs reflect efforts to accommodate language and culture instruction to the health care setting.

There are also less formal cross cultural educational resources such as workshops for continuing education credit, inservices, and brown bag lectures that focus on teaching about the Spanish language or culture of the Latino patients. On Febuary 13, 1996 the author attended the brown bag lecture by Dr. David Mata entitled "Cross Cultural Medicine: Bridging the Gap in Clinical Care" at the Oregon Health Sciences University (OHSU). This lecture was an effort to raise the cultural awareness of health care providers at OHSU about the Latino patient population in Oregon. The OHSU School of Nursing does not offer a course specialization in Transcultural Nursing and has not integrated a transcultural focus to its curriculum. ${ }^{14}$

\footnotetext{
${ }^{14}$ Graduate degree programs in Transcultural Nursing are available from four U.S. universities: Wayne State University in Detroit, University of Washington in Seattle, University of California in San Francisco and Los Angeles, and The University of Miami. Several other universities offer courses in transcultural nursing: Emory University in Atlanta, Medical College of Georgia, Florida Atlantic University, University of Toronto, University of Sydney and Cumberland College in Australia (Leininger, Transcultural Nursing Concepts 35-36).
} 
There are various local settings that offer classes for teaching Spanish to Health Care Professionals: Oregon Health Sciences University, uses the book Converse en Español: español para personal médico offered in one hour and fifteen minute sessions; Mount Hood Medical Center, uses the book ¿Qué Paso?; Portland Community College offers the continuing education course Hispanic Medical Beliefs and Culture through The Central Portland Workforce Training Center. This is a fourweek class and provides a total of 12 hours of instruction with each classroom session lasting 3 hours. The class text is the book ¿Qué Pasó? Under the category of "Special Interest" Portland Community College offers a class in Medicinal Herbs and Home Remedies. Portland State University offers non-credit classes on the principles of basic medical translation. Pacific University in Forest Grove has part of the Intercultural Communication Institute Library (ICIL) holdings temporarily for summer 1996. The programs at the University of Connecticut, University of Miami, and Rush Medical College are much more intensive classes than are currently offered in Portland metropolitan areas. In particular the University of Connecticut's Spanish 190: Language, Culture, and Health in Spanish Speaking Societies is a three week 8 hour per day immersion course.

Other resources available to health care providers interested in transcultural care include the professional journal articles and texts from 
the fields of nursing, cross-cultural medicine, and medical

anthropology. ${ }^{15}$ Unless the health care professional decides on a

transcultural specialization, receives inservices on transcultural issues, or

for some other reason has a marked interest in transcultural health care, it is unlikely that he or she will spend time investigating these sources. The aforementioned professional health care journal articles and texts do not teach the use of the Spanish language: they discuss transcultural patient care issues, using Spanish language terminology and concepts as points for discussion.

A common source of instruction is independent learning. In addition to English-Spanish medical dictionaries on the market, there are books sold specifically for teaching Spanish to health care professionals. The current books available to teach Spanish for the clinical setting that were examined by the author do not integrate the transcultural information and principles that have been addressed in professional health care journals. The quality of textbooks for teaching Spanish to health care providers is distinctly inferior to the high quality teaching materials in

15 Cecil Helman gives a definition:

Medical anthropology is about how people in different cultures and social groups explain the causes of ill-health, the types of treatment they believe in, and to whom they turn if they do become ill. It is also the study of how these beliefs and practices relate to biological and psychological changes in the human organism, in both health and disease.(1) 
related fields. Intercultural communication, cross-cultural medicine, medical anthropology and transcultural nursing texts introduce cultural information that is sorely lacking in textbooks for teaching Spanish for health care professionals. Texts for teaching Spanish to health care providers do a poor job of presenting cultural issues that affect Latino health care. Detailed information based on cross-cultural observations and investigations should be included in the Medical Spanish curriculum.

The author has examined many of these Spanish-for-health careproviders texts. (See Selected List of Works Consulted) The cost of these books reviewed ranged from $\$ 3.95$ to $\$ 29.95$. From these texts reviewed, the author has chosen thirteen different lesson examples to illustrate the methods of presentation used. The majority of the lessons are based on English to Spanish translations. Most often in these Medical Spanish or Spanish-for-health care-professionals texts are translations of medical history questions, translations of technical information, or pedagogical exercises. They are organized or have indexes so that the information can be cross referenced from one language to another.

The translated material is usually presented side by side on the same page in column form. In other instances the Spanish information is set up on one page and the English translation on the opposite page. An example of the column form as seen in the book ¿Qué Pasó? is as follows: 
How long have you had the ¿Por cuánto tiempo ha headache? tenido el dolor de cabeza?

(Kantrowitz 13).

Other books provide several paragraphs, pages, or chapters of reading in Spanish which then are translated into English. This information is presented according to body systems or specialty areas of interest. The book Medicina y Servicios Médicos: lecturas y vocabulario en español provides an example:

\section{Capítulo 6}

\section{EL OIDO}

El oído no tiene que ver solamente con la percepción auditiva, sino también con el equilibrio. Muchas personas .... (Schmitt 52)

This same text provides rather extensive lists of cognates and various forms of quizzes to test comprehension of what is read in Spanish. An example of the lists is as follows:
el potasio
la glándula
pulmonar
la retención
la hormona
curar (Schmitt 121)

Some texts are intended to stimulate conversation. The book Medical Spanish: A Conversational Approach briefly presents grammatical concepts at the beginning of each chapter and shortly thereafter provides structured questions so as to elicit the use of a particular grammatical 
form. For example:

Grammar

PRESENT INDICATIVE OF THE IRREGULAR VERB ESTAR (TO

BE)

yo estoy lam

él/ella hestá he is

usted (Ud.) está you are...

Answer the questions using the cues.

¿Dónde está el médico?

Where is the doctor?

El médico está en la clínica.

The doctor is in the

clinic.

1. ¿Dónde está la paciente?

- room

(DiLorenzo-Keaton 34)

This same book presents different forms of two column dialogue scenarios, for example:
A: ¿Qué piensa Ud. de la comida del
A: What do you hospital?
think of the
hospital food?
B: No me gusta.
B: I don't like it.
(DiLorenzo-Keaton 91)

The quick reference cards Speedy Spanish for Medical Personnel provide translations and offer an informal pronounciation guide for the vocabulary 
and phrases. Examples of vocabulary includes:

$$
\begin{array}{rr}
\text { testicles. . . (los) testículos } & \text { urine. . . (la) orina } \\
\text { tehs-tee-koo-loos } & \text { oh-ree-nah }
\end{array}
$$

An example of phrasing includes:

$$
\begin{array}{lr}
\text { I am ill. } & \text { I am nauseated. } \\
\text { Estoy enfermo(a). } & \text { Tengo el estómago revuelto. } \\
\text { eh-stoyehn-fehr-moh(mah) } & \text { tehn-goh ehl ehs- } \\
& \text { toh-mah-goh reh- } \\
& \text { vwehl-toh }
\end{array}
$$

(Baja Books, Anatomical Terms)

The book Spanish for Health Care Professionals presents short case scenarios followed by practice dialogues and vocabulary using an informal method to guide pronounciation. An example includes:

$$
\begin{gathered}
\text { Admissions } \\
\text { Admisiones } \\
\text { (ahd-mee-see } \bullet \text { 'oh-nehs) }
\end{gathered}
$$

The Espinoza family have been in this country for less than a year and they've suddenly ...

\section{Greetings}

Saludos (sah-'loo-dohs)

Do you speak English? ¿Habla inglés? ('ah-blah een'glehs) (Harvey 44) 
The book CommuniMed: Multilingual Patient Assessment Manual includes a list of 46 numbered items. Most are health assessment questions and a few are instructions or commands. The Spanish is on one side of the page and the English equivalent is on the opposite page. The book is designed so that the Engish-speaking health care provider can find the corresponding translation to health history questions, commands or instructions by matching the corresponding numbers of the items translated. The translations can be pointed out to the patient. The patient, in turn, is to read the printed responses in Spanish and point out a response. The answer chosen in Spanish by the patient can then be matched to the English translation which the monolingual English-speaking health care provider can understand. An example of the Spanish translation that is shown on the left page is as follows:

5. ¿Sufre usted de algunas de las enfermedades siguentes?

Elija todas las que sean apropiadas.

1. Problemas cardíacos

2. Problemas pulmonares

(Kelland 264)

The right page has the following translation:

5. Do you have/are you suffering from 
Pick one or more.

1. Heart problems

2. Lung problems. . .

(Kelland 265)

The book English and Spanish Medical Words and Phrases is a compilation of health assessment questions and other items of interest which are translated on the same page. For example:

Medications

Do you take any medications?

- Prescription?

- Over the counter?

- Other?

(Loeb 114)

On the other half of the page is the translation. For example:

Medicamentos

¿Toma Ud. medicamentos?

- ¿De receta?

- ¿Sin necesidad de receta?

- ¿Otros?

(Loeb 114)

The book Manual for Relatively Painless Medical Spanish briefly presents grammar principles and vocabulary. The exercises consist of English 
words or phrases and the Spanish equivalents on the same page. There are many translation exercises and practice dialogues. Many of the structured exercises consist of fill-in-the-blank sections. For example:

Translate into Spanish:

I need the doctor. Yo necesito

I need the nurse.

I need the surgeon.

Do you need the doctor? ¿Necesita usted $?$

(Rajkovic 45)

Of the numerous texts reviewed that were sold under the category of "Spanish for health care professionals," the book by Harvey, Spanish For Health Care Professionals, had a section of a few entries entitled "Dos Culturas." This section presented superficial comments on cultural information. An example of the one that yields the most cultural information is as follows:

Millions of people in the United States do not believe in pharmaceutical drugs. Many Hispanics still rely on medicinas caseras or home remedies. . . You may want to read up on such methods, not only to learn more about their culture, but also to find out if what they are taking at home has any real medicinal value. (Harvey 124)

The text Spanish for Medical Personnel by Janet E. Meizel, includes a five 
page section on folk-medicine practices. This also demonstrates an initial effort at acknowledging and incorporating cultural instruction into Spanish language training, instead of merely translating health assessment questions and the names of health conditions into Spanish.

Two of the texts reviewed--Spanish for the Medical Professions by Ralph Kite, which is of the Destinos Spanish instruction series, and Spanish For Medical Personnel by Jarvis Lebredo--included interspersed short segments, from a few sentences to up to a paragraph, written to explain cultural details relevant to the subject matter of the chapter. An example of this in the book by Ralph Kite is as follows:

Nota cultural: La papa se originó en el Perú en la época de los incas. Los españoles la llevaron a Europa en el siglo XVI (1500s). Hoy es un alimento básico en todo el mundo, especialmente en la región andina (Andean). (28)

An example from a paragraph from the book by Jarvis Lebredo is as follows:

The use of health care services by Hispanics depends greatly upon socioeconomic status and type of employment because these affect access to comprehensive and preventive medical care. Data from the Hispanic Health and Nutrition Examination Survey indicates . . . (79)

The section of Notas culturales was found to be one of these books' 
strengths. These books contain other positive features geared for the classroom setting: dialogues that better simulate true-to-life patientprovider conversations; ample vocabulary; cognates; a few medical applications to fill out in Spanish; situations in which students are required to interview each other in Spanish; exercises that require students to improvise and act out situations; sentence and dialogue completion exercises; taped dialogues for listening comprehension exercises; and reading comprehension materials incorporating health care topics. These last two books seem to be the most useful for stimulating active participation from students. The books, especially the one by Jarvis Lebredo, present clinically useful information, and the exercises call upon students' creativity in practicing the language. These books in particular start to approximate the kind of cultural information that needs to be included in Spanish language instruction for the clinical setting.

The author found a noteworthy handbook under the category of "Transcultural Nursing" entitled Pocket Guide: Cultural Assessment by Elaine Geissler. This book is alphabetically organized according to name of country. The index is a reference to one-to two-page sections containing health-related and non health-related facts, including some cultural characteristics of persons from these countries. ${ }^{16}$ Not all of the

${ }^{16}$ Information provided in the section contain the following categories: country; location; major languages; ethnic groups; major 
Spanish-speaking countries are mentioned. This pocket guide is very useful as a reference for quick cross-cultural health-related information. However, the author thinks that more information could be given, especially in the section of ethno-medical health care practices. The ideal is to find a middle ground between the type of information published in the transcultural sciences journals and the specialized language texts for teaching Spanish to health care professionals.

As seen from these examples, current Spanish-for-health careprofessionals texts provide basic Spanish language instruction but the cultural information on issues that affect patient's health care is either non-existent or poor. Because these texts seldom acknowledge the use of folk medical practices, there is a void in the Medical-Spanish information available. English-speaking health care providers often search for books from which to study on their own and at their own pace. They face a dilemma because they are not fluent in Spanish, and they cannot judge the quality of the instructional books on the market. Medical-Spanish language students may learn vocabulary and phrasing useful to the clinical setting but at the same time be at a loss for understanding "patient behavior and the way the patient perceives the

religions; predominant sick care practices; ethnic race specific or endemic diseases; touch practices; preceptions of time; birth rites; food practices and intolerances; national childhood immunizations; other characteristics; bibliography. (Geissler 52-53) 
illness or health situation" (Tripp-Reimer 78). Relaying of biomedical

information or treatment instructions in Spanish remains superficial and is not adequate if the cultural context of the patient's illness is not understood. 
III. Current Tools For Improving Communication Between Patients and Health Care Providers

Transcultural health care research clearly signals the need for health care professionals to develop both linguistic ability as well as cultural competence for working with Spanish-speaking patients in the medical setting. Transculturally focused health care education--including specialized Spanish language and culture instruction--helps to "prepare graduates who are 'culturally sensitive, knowledgable, and competent'" (Carpio 4). Health care professionals who speak Spanish and are familiar with the implications of culture on the Latino patient's health care beliefs and practices will be better equipped to provide culturally relevant services.

There can be a cultural rift between patients and health care providers with regards to the use of biomedicine and folk medicine. The number of persons who actually use folk medicine and the reasons for the use of folk medicine make it a significant enough topic for health care providers to be familiar with these practices. The incongruity between biomedicine and culture-bound syndromes or folk illnesses often causes a negative reaction by health care providers. Robert Weclew explains that health care providers "often put off Chicano patients by scoffing at folk ideas (concepts inherent in curanderismo) or otherwise violate cultural etiquette" (148). Clarissa Scott reminds health care providers that the 
imposition of ethno-medical ideas works against provider-patient rapport:

"To try to change a deeply rooted health belief either by ridicule or by treating it as unscientific may not only fail but may also alienate the patient" (531). A therapeutic approach would not impede communication.

Health care personnel can try to bridge this dichotomy between modern biomedicine and folk beliefs by keeping in mind the possibility that patients have alternative health care perspectives. Gonzalez-Swafford reminds health care practitioners that patients have their "own perception of what's wrong" (29) and recommends that "listening carefully to what already has been tried--and not tried--at home to alleviate symptoms" be a vital part of assessing a client (30). Scott recommends "[using] a treatment plan which shows understanding and respect for the patient's beliefs and which builds on these in a positive way" (531). This is necessary because if a medical treatment is incongruent with or contrary to a client's well engrained cultural beliefs and self-care practices, it is much less likely that the client will follow through with the medical treatment regimen. Each patient's cultural orientation with regards to health care beliefs and practices should be assessed. The opportunity for assessment is inherent to clinical interaction with patients. When there is very limited time for contact with the patient--as is the reality of most clinical situations--a partial cultural assessment is most useful. The most 
basic information to determine is whether the recommended medical treatment is culturally acceptable to the patient and if it is likely that it will be followed through by the client.

Cultural assessment has been written about extensively in professional health care journals and texts. There is a wide variety of cultural assessment tools available. The degree to which cultural assessment tools are practical for use in the clinical setting--that is, whether the questions are phrased so that the patient can understand them--depends on the complexity and abstractness of the questions. There are examples of highly abstract and theoretic tools available in the literature. ${ }^{17}$ The tool by Janet Rosenbaum uses a less abstract approach which lists questions. Toni Tripp-Reimer provides questions that are formulated to elicit "problem-specific cultural information" (81). The author favors the assessment questions by Chesney, who suggests a health care practitioner determine:

What they think is wrong? or what they think is causing the illness?; what they think might help treat the illness?; what

17 Madeleine Leininger published a highly theoretic and abstract outline of areas to be assessed. A slightly less theoretical topic outline was published by Janis Bellack. These tools do not provide examples of which questions to ask. The health care provider is to formulate questions as is appropriate and opportune. Very lengthy and thorough tools are Giger and Davidhizar's Transcultural Assessment Model and Bloch's Assessment Guide for Ethnic/Cultural Variations. 
the patient has done to treat the illness?; what the patient would have done to treat the illness if a physician were not available? (573)

For a quick orientation to the use of cultural assessment tools, another easy-to-use tool that is recommended to the reader is found in Angeline Bushy's, "Cultural Considerations for Primary Health Care: Where do selfcare and folk medicine fit?" If such assessments can be done in a manner that is non confrontational and non judgmental, then the patient will be more at ease about sharing such information.

Patients often are reticent about revealing their health care beliefs and practices. Patients may mention details to the health care providers which are clues to their beliefs and self-care practices. Kreisman recommends that providers be "aware of subtle signs which indicate that a patient's concept of cultural illness is conflicting with a traditional therapeutic program" (82). As Robert Ness has noted "[P]eople who believe in folk healing may be very reluctant to discuss anything but physical complaints or vague feelings of nervousness because of fear of being misunderstood, not taken seriously or ridiculed" (1481).

In the 1993 article "Developing Therapeutic Relationships in Multicultural Settings", Sandra Rankin also shows strong support for the observation that Latin American patients often do not disclose their use of folk remedies: 
A national survey has disclosed wide use of unconventional therapies. Furthermore it found that $72 \%$ of the respondents did not tell their physicans they used such alternative treatments, including traditional ones (i.e., those that are culture-specific, such as Hispanic). . . . The ensuing conflicts between ethnic and allopathic medicine can result in low rates of utilization of allopathic care among some patients. . . (826)

This is a striking statistic. It supports the author's supposition that the majority of Latino patients are guarded about sharing information with regards to their folk health care practices.

The aforementioned tools are useful for conducting patient assessments. They are also instrumental in drawing attention to cultural issues that should be addressed in Medical Spanish or Spanish-for-health care-provider courses. 
IV. Cultural Considerations in Patient-Provider Communication

Health care personnel will have gaps in communicating with and understanding Spanish-speaking patients if they do not understand the patients' culture. The integration of cultural concepts into Spanish language instruction is an ideal situation in which to raise cultural awareness. This investigation will move the discussion to topics that influence Latino patient's health care behaviors. Some of the health care related issues include culture-bound illnesses or folk illnesses and alternative treatment practices; social support networks; familism; authority structure; locus of control; fatalism; and communication style.

Both patients and health care providers hold deeply engrained beliefs about health, illness and treatment. They are "culture-bound" in their views (Maduro 868). While there are patients familiar with aspects of biomedicine to some degree, the majority of patients hold a variety of nonprofessional or lay ideas about health, illness and treatment. Some "lay" explanations of health and illness are personal, idiosyncratic and creative. Other patients' beliefs have a strong foundation in traditional folk medicine. The latter is the case when dealing with some of the Latino patient population. Latino patient's health care beliefs and practices influenced by traditional or "folk" beliefs are different from the standard biomedical approach. Understanding the client's non biomedical perspectives is necessary because they influence the health care decisions 
that the patients make and affect their self care. This also reflects in the patient's "adherence to treatment recommendations" (Engel 125).

Most non Latino English-speaking health care personnel are not familiar with health and illness concepts unique to the Latino culture. Greatly due to educational background, health care professionals are culture-bound to biomedical health care perspectives. ${ }^{18}$ Biomedicine is the dominant treatment approach in the U.S. Health Sciences education places emphasis on the principles of biomedicine and seldom addresses alternative health, illness and treatment belief systems. Alternate medical belief systems and treatment measures are considered secondary, adjunctive, or belonging to the realm of folk medicine or ethno-medicine by practitioners of allopathic ${ }^{19}$ medicine.

Through the educational process the health care professional's conceptualization of health and illness "takes on a more abstract and scientific character" and experiences a "removal from the lay mode of thinking." (Spector 7) The biomedical concepts and jargon may be

${ }^{18}$ A definition of the biomedical approach is "pertaining to those aspects of the natural sciences, especially biologic and physiologic sciences, that relate to or underlie medicine" (Steadman's Medical Dictionary 171).

19 Allopath is defined as: "One who practices medicine according to the system of allopathy" (Steadman's Medical Dictionary 44). Allopathy is defined as "substutive therapy; a theraputic system in which a disease is treated by producing a second condition that is incompatible with or antagonistic to the first" (Steadman's Medical Dictionary 44). 
"beyond the linguistic system" of the average patient (Putsch 3344). In other words, the patient may not understand the technical terminology and jargon. Health care personnel will have to provide services for and communicate with Latino patients from a range of socio-economic levels. Greater differences in conceptualizations will be found among patients who have a lower level of education. A key to clearer communication with patients is to be "able to perceive the concept of health [and illness] in the way that a vast number of health-care consumers may perceive it" (Spector 7).

Latino patient's understanding of biomedical terminology and acceptance of biomedical practices will vary according to education, and socio-economic level as well as other factors. As Linda Haffner points out, "Highly educated and affluent Latinos have attitudes and beliefs about health care reasonably comparable to those of similarly educated and wealthy Americans" (255). Therefore, some of the Latino population will believe in the practice of biomedicine more than others. Biomedical information and instructions can be explained in Spanish to a patient; however, this does not assure that the patient will fully accept this explanation in lieu of their non professional beliefs.

Health care providers will occasionally find that Latino patients describe folk illnesses or culture bound syndromes which are "illnesses that are not known or recognized by modern medicine" (Moore 127). The 
terminology for the folk illnesses and home remedies often is outside conventional medical terminology as they do not have a scientific equivalent in modern medicine. The terminology for these folk illnesses cannot be understood by non Latino providers unless they have had some type of transcultural education. There are numerous folk-medical concepts and illnesses that have been written about in the professional medical, nursing and anthropological journals. ${ }^{20}$ The preferred treatment for culture-bound illnesses is the use of home remedies, folk remedies, and folk healers (Chesney 572). Simmons writes, "In many cases priority is given to home therapy and the folk specialist, while the doctor is consulted as last resort" (68). Chesney states, patients "may consult a folk healer before consulting a physician, may consult a physician before consulting a folk healer, or may use folk healers and physicians simultaneously" (571. See also Ness 1478; Simmons 69; Trotter 114.)

Families may try to treat the sick person at home before looking for outside assistance. Traditional home remedies or folk-remedies can

20 Examples include: Hot and cold theory of disease, empacho, susto, asoleo, ajito, bilis, caida de la mollera, chincual, mal aigre [sic], mal aire, pasmo, dolor del aire, mal de ojo, malefacio, el daño, mal puesto, mal de latido, celos, melarchico, pujo, bilongo, chipleza, nervios, cuarentena, antojos, ataque, tirisa, suspendido, etc. It is interesting to note that the Italian and Greek cultures have concepts similar to mal de ojo which are the Mal'occho (See the article by Alan Kraut) and Matiasma (See the article by Toni Tripp-Reimer). 
include the use of herb teas ${ }^{21}$, herbal preparations ${ }^{22}$, or over-the-

counter medications. Ford notes regarding the common use of herbal medicines,

Just as many Anglo-Americans have aspirin, cold tablets, stomach remedies, cough drops, rubbing alcohol, and Bandaids in their medicine cabinets, many Hispanics keep rose petals (Rosa de Castilla -- $\underline{\text { Rosa }} \mathbf{s p p . )}$ for sore throats,

21 "The 25 most common remedios caseros" of the study conducted by Robert Trotter are as follows:

manzanilla (camomile) Matricaria chamomilla L.; savile (aloe vera) Aloe barbadensis Mill.; ruda (rue) Ruta graveolens L.; yerba aniz [sic] (anise) Pimpinella anisum L.; verba buena (mint) Mentha spicata L.; estafiate (worm wood) Artemisia mexicana Willd.; hojas de naranjo (orange leaves) Citrus aurantium L.; albacar [sic](sweet basil) Ocimum basilicum L.; oregano (oregano) Monarda methaefolia Graham; ajo (garlic) Allium sativum L.; pelos de elote (corn silks) Zea mays L.; canela (cinnamon) Pulchea orodata (L) Cass; romero (rosemary) Rosmarinus officinalis L.; borraja (borrage) Borajo officinalis L.; cenizo (purple sage)Leucophyllum texanum Benth.; nopal (prickly pear cactus) Opuntia sp.; Rosa de Castillo [sic](rose) Rosa centifolia sp.; salvia (sage) Salvia leucantha Cav.; hojas de mesquite (mesquite leaves) Prosopis glandulosa Torr.; marijuana (marijuana) Cannabis sativa L.; nogal (pecan) Carya illonoensis Koch; comino (cumin) Arracacia atropurpurea Benth. et Hook; golondrina (swallowwort) Euphorbia prostrata Ait.; sacate de limon (lemon grass) unidentified local plant; e/ azajar [sic](orange blossoms) Citrus aurantium L. (109)

In the above citation the correct spelling of several herbs is as follows: anis, albahaca, Rosa de Castilla, and azahar (Cabello, Thesis Commitee).

${ }^{22}$ The author recommends the book Las Yerbas de la Gente: A Study of Hispano-American Medicinal Plants. This book contains an enormous botanical list of herbs and their curative properties. 
manzanilla (Matricaria spp. or Athemis sp.) for stomach upsets and colds, oshá (Ligusticum Porteri) for cuts, inmortal (Asclepias capricornu) for various ailments, and alhucema (Lavandula sp.) for infant's stomachaches. . . . (5)

It would be beneficial for health care providers to be familiar with some of the most common herbal infusions and preparations used by patients of the Latin American community. The mixing of prescription medications with herbal remedies or the use of several herbal remedies at once may be contraindicated due to incompatibilities which may cause various adverse effects or contribute to polypharmacy. The Latino population may be more prone to viewing herbs as being distinct from drugs. In differentiating the two, "the distinction is more typically between natural/organic medications and synthetic or semi-synthetic ones" (Farr, Thesis Commitee). The fact is some herbs are powerful medications which, if used improperly have adverse side effects. An article in The Oregonian, "Healer treats mind, body, spirit," discusses this: "Taking herbs that clash with drugs can send patients to the hospital. . . It can be a real nightmare for physicians to figure out why people react the way they do to medication" (Gonzalez B3).

Patients may choose to administer home/folk medicine--use home remedies--for self-treatment or the treatment of ill family members. In addition, some patients may consult with folk medicine specialists/folk 
healers. As Hernández notes, "regional healers are consulted only after family healers and their remedies, which include administering various herbs made up in various teas coupled with prayer or treating for one of the folk illnesses, have been used" (268). There are several types of folk healers. ${ }^{23}$ Orque explains one type of folk healer: "The curandero (male) or curandera (female) is a folk healer who cures by using rituals, prayers, herbs, massages, and the laying on of hands" (130). In a study by Robert Weclew, the Latin American patient population interviewed was of the opinion that curanderos or folk-healers practiced according to "suggestion, practical advice, herbs, folk remedies, as well as various prayers and religious rituals" (151). Maduro provides a comprehensive description of the practice of curanderos: "[C]uranderismo is syncretic, eclectic and holistic; it is a mixture of beliefs derived from Aztec, Spanish, spiritistic, spiritualistic, homeopathic and modern 'scientific' medicine" (869). Ruiz provides a list of treatment methods used by another type of folk healer: "(Santeros) use such treatment approaches as prayer, animal sacrifice, special baths, perfumes, oils, candles, herbs, weeds, plants and the like" (90). There is much variety among folk practitioners and the treatments that they provide.

${ }^{23}$ Some of the many names for folk medical practitioners are parcheros, espiritualistas, sobadores, yerbero, parteras, brujos, el que sabe, la que sabe, santeros, espiritistas, curanderos, curanderas. 
Research is inexact as to how often and to what extent patients practice self-care according to folk-medicine. In his research Robert Weclew notes: "a substantial minority of [Latin American] respondents felt that the greater the degree of education, affluence, and acculturation, the more likely that a client would shun the curandero and turn to the mental health center" (152). Tripp-Reimer found that a variable which influences the use of folk medicine is the difference between generations, differences among first-generation, second-generation and third-generation immigrants (Folk-Healing Practice 97-101). A 1987 study by Chesney revealed that, of the target population studied, "68 percent of [Mexican] families utilize both conventional and folk medicine" (571). In addition, the manual by COSSIMO notes:

In fact, HHANES reported that fewer than 4 percent of Hispanics used a folk healer in a 12-month period. Another observer found that 9 percent of the Hispanics using a clinic in a low-income neighborhood in Los Angeles reported having ever used a curandero/curandera . . . One observer did find the use of healers to be a persistent though ancillary health care system among Mexican Americans in Southern California. (60)

Despite the fact that not all Latino patients use or agree with the use of home remedies or folk-medicine, there are several reasons why patients 
may choose to do so. A practical reason is that there is a significant segment of the Latino population in which folk medicine may be used as an alternative by persons who cannot pay for medical care. Another facet keeping folk medicine in use is that some undocumented immigrants do not want to come into contact with the medical system. As Dehn notes, "health care is a severe problem for people who fear deportation and alternate forms of cure are seen as preferable" (14).

For some patients, alternative folk-medicine treatments can be sources of care that are "less expensive and more trusted [than biomedicine]" (Dehn 12). There is incongruity between modern medicine and folk medicine. Some patients are of the opinion that:

the doctor's remedies are ineffectual for certain [folk] illnesses or may actually aggravate them because the doctor does not "know" these illnesses or does not "believe in them" and [therefore these patients] prefer to use folk medicine. (Simmons 67)

Some persons choose to use folk medicine because they are disappointed with the biomedical treatment; they "turn to folk healers if they feel that ... the medical system has failed to cure or eliminate [an illness]" (Trotter 114). Others use home remedies and folk medicine because it is part of the culture and it is a method they have learned to use. Rubel writes that folk illnesses and their treatment have "become 
highly symbolic of a traditional way of life" (814). Hernández comments about the extent to which it represents traditional culture:

Anglos are believed to be immune to such ailments, merely being afflicted by one is a means of cultural identification with la raza. To accept the diagnosis and to cooperate in the treatment are a declaration of acceptance of the conservative Mexican-American world view. (276)

I will limit the discussion of folk medical concepts to the following three: the hot-cold theory, empacho, and susto. They are representative of frequently occurring non-biomedical health care concepts. The hot-cold theory, more prominent than the others, will be discussed at greater length.

Folk beliefs and folk medicine periodically appear during patient care and, therefore, providers need to be aware of these concepts. There are patients who strongly believe and practice self-care ${ }^{24}$ according to precepts of the hot-cold theory and who base their health care decision and practices accordingly (Gonzales-Swafford 29). Conversely, there are many Latino patients who are either unaware of its existence or who are

24 Self-care is defined as:

the practices a lay person uses on his or her own behalf in health promotion, disease prevention, and in disease detection and treatment .... [S]elf-care encompasses a wide range of universal skills, which include rituals, remedies, and folk medicine, to retain and restore health. (Bushy 11) 
very skeptical and altogether discount its validity. Harwood notes that there are also other patients in which "the culture pattern may still survive in attenuated form" (1157).

The hot and cold theory of illness causation and treatment is a traditional model based on the concept of balance. Despite overt and fundamental differences between modern medicine and the hot-cold theory, both have the concept of balance in common. Helman explains about the concept of balance in biomedicine:

Modern physiology does include numerous examples of diseases that are caused by a deficiency, or excess, of certain substances in the body, such as hormones, enzymes, electrolytes, vitamins, trace elements and blood cells, which can be corrected by replacing the deficient substance, or counteracting excess. (21)

These biomedical ideas of balance among body systems differ from the type of balance spoken about in Latin American folk medical theory of hot-cold used for self-care. ${ }^{25}$ The premise of the hot-cold theory is that

25 Despite the predominance of biomedicine's focus on treating the body, recent professional literature reflects a greater concern for a holistic treatment approach. A holistic approach to medical care takes into account not only a patient's physical state but also the patient's psychological, social and spiritual well being (Modesta 125), all of which are integral parts to the wholeness of each individual. It is interesting to note that as modern biomedicine advances, it reformulates and reinstates variations of prior existing ideas at the same time that it is future oriented, 
illness is caused by an imbalance of "hot"and "cold" substances in the body. Health is maintained by keeping a balance between internal and external elements that affect the body, a concept which developed from ancient Greek medicine:

According to this classical pathology, the body is regulated by four humors, or fluids, each of which is classified by a combination of hot or cold with wetness or dryness (bloodhot and wet; yellow bile--hot and dry; phlegm-cold and wet; black bile-cold and dry. These humors must be properly balanced to maintain health and prevent illness. (Orque 125) An excess or lack of a certain humour is thought to bring on illness. From this, the Latin American hot-cold theory evolved to emphasize the balance

promoting wellness. As Burk states, "A holistic model of primary care encompasses health promotion, health maintenance, and disease prevention. Cultural sensitivity is integral to the success of such a model" (38). Meeting patient's spiritual and social needs is not a new concept. A similar holistic idea is present in traditional Latin American folk medical perspectives. From a traditional holistic point of view a patient may perceive that an illness is "a complex physical-social-spiritual problem involving not only themselves but also their family, social networks and their relation with the spirit world" (Ness 1481). As Robert Weclew comments, "The curandero and his patients view illness in a religious and social context, not in the medical-scientific one of the Anglo society" (146). Health care journals and texts are beginning to reflect a push towards transcultural health care education which is part of promoting the holistic care of patients. One example of this is the Neuman Systems Model which "explicitly incorporates culture. The person is described as a composite of physiological, psychological, developmental, spiritual, and sociocultural variables" (Capers 20). 
of hot and cold in particular.

The hot-cold theory is a complex traditional conceptualization of disease causation. It affects prescribed treatments which range from food to herbs, medications, and other substances. The origin of the LatinAmerican hot-cold theory of folk medicine is ancient. Studies trace it back to folk medicine practices of China and India which were investigated by the Greek physician and scholar, Hippocrates (460-377 B.C.), who developed the humoral theory of illness causation and treatment. Later Galen (131-201 A.D.), a Greek physician in Rome, reintroduced the teachings of Hippocrates which spread throughout the Roman and Islamic Empires over hundreds of years. In the 800 's A.D. Moorish scholars translated Galen's works on humoral medicine into the Arabic language and expanded the theory. Under Moorish domination, humoral medicine was introduced to the people of the Iberian Penninsula. Eventually the Spanish and Portugese brought these humoral European medical practices to North, Central, and South America during the time of colonization in the 1500 s and 1600 s. At this time Spanish Catholic priests provided medical attention to the indigenous and mestizo populations of the Americas based on the training and references they used from humoral medicine. The indigenous populations already had advanced knowledge of herbal medicine. The Inca, Aztec, and Mayan civilizations had knowledge of "medicinal plants. . . that had curative 
properties" (Branch 53). The Inca and Aztec civilizations had similar humoral concepts prior to the colonization by the Europeans. A syncretism occurred between indigenous folk medicinal herb practices and the use of European humoral medicine. This syncretism still influences current folk medical practices in Latin America. Renaldo Maduro goes so far as to say: "Clearly the notions of Galen and Hippocrates are still alive and are a basic part of Latin American folk medicine" (871).

Discussion of the hot-cold theory in the professional health care literature is abundant. An article by Richard Currier describes the widespread existence of this theory in Latin America:

The hot-cold syndrome in Latin America has been reported for Mexico. . . for Mexican American communities . . . for the Guatemalan Highlands. . . for coastal Colombia . . . for the Colombian Highlands. . . and for coastal Peru and coastal Chile. . . (253)

Its existence among the Puerto Rican community has also been investigated (Harwood 1153-1158; Spector 219-220). Many articles have been written about its existence among the Mexican and MexicanAmerican community. Harwood has done research on which persons are more apt to practice self-care using home remedies based on the hot-cold syndrome. The parameters used were according to age and education. Harwood's conclusion reveals: 
The elderly and recent adult migrants who never completed high school are most likely to espouse the hot-cold system openly and consciously to weigh theraputic decisions within its framework. ... People who have been born in the States, on the other hand, or who have received most of their primary or secondary education here are not likely to express agreement with the hot-cold system openly. They may, however, defend those parts of it which agree with standard medical practice. (1157)

Harwood further notes: "[T]he process of acculturation through the generations creates subtle variations in the way hot-cold beliefs manifest themselves" (1157). Self-care practice according to the hot-cold theory is variable among the Latino patient population. However, its existence makes it a topic worth exploring.

Folk remedies and illnesses are classified as hot--caliente--and cold-fresco or frio. To restore the balance of hot and cold in the body folk remedies are administered in the form of foods; beverages such as herbal teas; medicines such as emetics, diuretics or cathartics; ointments; compresses; physical manipulations of different sorts, as well as other things. Folk remedies are assigned calidades or qualities of hot and cold which are thought to affect the hot-cold equilibrium of the body. This quality assigned to a remedy is not due to its physical temperature, but 
rather due to the effect the substance is believed to have in the body (Harwood 1154; Antony-Tkach 426). "[l]f an illness is classified as hot, it is treated with a cold substance. A cold disease, in turn, must be treated with a hot substance" (Ailinger 634), Helman explains:

[T]here are "cooling" and "heat-producing" foods, which are used to reduce the excess of a humour; hot foods can cause excess bile, and thus illness must be treated by a diet of cold food and other [cold] medication. . . . In illness, health is restored by re-establishing the internal temperature balance by exposing oneself to, or ingesting, items of an opposite quality to that believed to be responsible for the illness. (19) Research has documented that there is much variation to the hot and cold qualities assigned to illnesses, foods, herbs, medications and other substances (Antony-Tkach 426; Currier 253). Karen Ford writes "[C]lassification of ailments and herbal remedies into hot, cold, or in between categories may be idiosyncratic to the extent that whether an item is hot or cold may vary from one specialist [curandera] to another ..." (6) Harwood disagrees with this:

While there is general agreement about the assignment of foods and medicines to the categories. . . the system allows for variation as well. In general, a person may categorize a food or medicine differently from the norm if it 
idiosyncratically produces physical symptoms which are typically classified as hot or cold. (1154)

Gonzalez-Swafford takes a more pragmatic view of why variation is present among the Mexican-Americans population in her study:

Although there is no general agreement as to which foods and diseases are hot or cold, each Mexican-American family has adapted and refined its beliefs to best fit the foods available to it and the illnesses by which it is most often plagued. (30)

Researchers have differing opinions about why such intracultural variation has developed. Ingham writes that the intracultural variation encompasses the mixing of European and indigenous cultures: "there are numerous regional variations that have doubtless resulted from the syncretism with local Indian cultures" (86). Gonzalez-Swafford emphasizes that the principle of balancing food exists and is retained despite the differences in categorizations. Currier draws the conclusion that "what is important is only that foodstuffs be assigned a calidad. . . . [I]t makes little difference which particular substances are classified as hot and which are cold" (253).

Such commentary is a modern psychological interpretation for the usefulness of the hot-cold theory. Some modern interpretations are based on the idea that the hot-cold syndrome is a metaphor for the balancing of 
intimacy and withdrawal in human relationships (Currier 252; Ingham 76). Intimacy and warmth in intrapersonal relationships such as in family, extended family or compadrazco relationships is a significant part of the Latino culture. Another interpretation for the symbolism of the hot-cold theory is noted by Rubel who states that it is reflective of and can be "attributed to tension caused by contradictory role demands made by society upon the individual" (Rubel 799). Interpretations of the symbolism behind the hot-cold theory can be viable as a reflection of an actual cultural and interpersonal phenomenon.

As was mentioned earlier, not only are foods categorized as hot and cold but medications are also. If a patient thinks he or she has a hot illness, then a cold or cooling treatment will be sought; a hot or warming treatment most likely will be rejected for a warm or hot illness condition and visa versa. Gonzalez-Swafford speaks of cold illnesses and treatment:

Cold diseases are caused by excessive exposure to cold environmental elements or by overconsumption of cold foods. Cold diseases produce invisible symptoms such as pain and immobility. . . . Treatment for cold illnesses is just the opposite of that for hot-ingesting hot foods, using warming herbal teas or applying warmth to a body area. (30) Patients' self-care may reflect their belief in "re-establishing the internal 
temperature balance by exposing oneself to, or ingesting, items of an opposite quality to that believed to be responsible for the illness" (Helman 19).

Several researchers have noted this with the Latin American's use of vitamins, penicillin, aspirin, and other medications (Flaskerud 432; Harwood 1154; Maduro 871). Antony-Tkach notes:

A common belief is that prenatal vitamins are a "hot" food and thus not to be taken during pregnancy because pregnancy is considered a "hot" state of the body. However, taking the vitamins with fruit juice, a "cold" food, cools down the vitamins. (426)

Gonzalez-Swafford comments: "Aspirin and penicillin, for example, are both thought to be hot, and prescribing either of these drugs for a correspondingly hot illness such as a sore throat or fever would be against the client's beliefs" (30). Hartog describes the concept of neutralization in relation to taking medications "[S]ome cultures consider penicillin a "hot" substance and therefore will refuse an injection in case of a fever, but orange juice is a cold food [sic]. Penicillin given in orange juice might be acceptable" (914). If the provider can find out whether the client bases health care practices on the hot-cold theory, treatments or medications can be recommended in a more culturally acceptable manner. Otherwise, the treatment may be rejected by the patient due to the 
cultural "dissonance or incongruence" (Branch 73).

Treatments for common conditions, such as is prescribed for a common cold, becomes a bit more complicated when dealing with patients who believe in the hot-cold theory. Using a little creativity, the culturally sensitive practitioner can work around these differences in beliefs:

Suggesting orange juice, which is high in vitamin $C$, for a chest cold may not be appropriate if the client regards it [orange juice] as a cold food and therefore unsuitable. Chili, on the other hand, is also a good source of vitamin C, but is hot and more in keeping with a client's belief that equilibrium should be restored. (Gonzalez-Swafford 30)

Another instance in which the health practitioner needs to be aware of the patient's health beliefs is when a patient is taking a diuretic that depletes the potassium level of the body. If, for some reason, the patient modifies his or her diet and does not replenish the potassium through diet, the drop in potassium level in the body can be life threatening (Ailinger 634). As Harwood explains:

When prescribing a diuretic, routine medical practice is to encourage the patient to eat bananas, oranges, raisins, or other dried fruit in order to maintain the potassium balance .... . [A]ll these foods are considered cold or cool, 
when a patient contracts a common cold or other coldclassified illness, he will stop eating these potassium sources because they are contraindicated for his immediate [cold] condition. . . A way out of this dilemma might be for the physician to prescribe potassium ... as a vitamin land therefore hot). . . or to suggest foods rich in potassium (like coffee, cocoa, peas etc). (1155)

A simple case of a common cold usually is not life treatening, as it is a transitory illness, but if other variables are figured in such as the use of a diuretic, the situation becomes more complicated if folk practices are also involved. In other instances cultural knowledge can be even more crucial when dealing with a patient's condition. Consider the following:

"A baby with dysentery (a cool disease in Guatemala) may be denied water because the water is considered a 'cold' substance, and sick babies need to be kept warm" (Hartog 914). In the aforementioned situation treatment according to folk beliefs contributes to the rapidly worsening condition of the patient.

Folk beliefs can influence adherence to recommended medicine and diet. A patient may refuse a recommended diet or the food served (Spector 215). For instance, a woman who is menstruating, pregnant, or post partum is considered being in a "warm" body state (Currier 257; Anthony-Tkach 426), and this warm condition can have a bearing on her 
dietary choices. As Helman notes, "postpartum or menstruating women ... may avoid certain fruits and vegetables, which they classsify as cold and liable to clot their hot menstrual blood" (Helman 19). The belief of diet affecting flow of the locia or of menses is something that is not supported by biomedicine. This type of dietary restriction can lead to problems of a "diet deficient in vitamins" (Helman 19) or some other dietary insufficiency. For the most part, this cultural belief is unknown to the non-Latino health care practitioner. In keeping with the same case scenario, a patient might stop taking any medication that is categorized hot until their own "warm" illness condition passes. This can quickly drop the blood level of medications. In fact, according to folk beliefs, taking a hot remedy during a hot condition and vice versa can be dangerous. Implications of the hot-cold theory for medical care can be quite involved. A culturally aware health care provider will be familiar with the variation of folk practices used by patients.

In medical literature it has been documented that patients use potentially dangerous substances in home remedies without knowing that they are harmful. For example, "One remedy for gastrointestinal discomfort is mercury, which acts as a cathartic" (Anthony-Tkach 426). Other examples of dangerous home remedies include the use of greta and azarcón which are lead based, azoque or "elemental mercury" and anil, or 
"laundry bluing," all of which are poisonous. ${ }^{26}$ These substances are used as cathartics in the home remedies for the treatment of empacho which will be discussed below. A more common practice is the self medication with antibiotics. Antibiotics are available over the counter in many Latin American countries and are one of the many over-the-counter medications that have been incorporated into home and folk medicinal practices. Such unregulated use is thought to have contributed to the evolution of drug resistant organisms. A culturally aware health care provider who can communicate well in Spanish will be able to inquire about and understand the patient's self care practices. Ideally, the practitioner will be better able to work along with the patient to achieve a more effective medical treatment outcome.

The second Latin American folk-illness that will be discussed is susto or mal de susto. According to folk beliefs, it is an illness that cannot be cured by modern medicine (Simmons 67), but only by folk medicine. It has been labeled an "anxiety syndrome" (Kiev 69) and a

${ }^{26}$ The Multnomah County Health Department in Portland, Oregon administers a questionnaire to parents of children who have high levels of lead in their blood. First the questionnaire asks the general question of whether they use any home remedies. Then the questionnaire specifically asks about several common home remedies that have been identified in the past as sources of lead poisoning: "Azarcon [sic]; Liga; Alarcon [sic]; Greta; María Luisa; Rueda; Coral" (3). The correct spelling for the words above are Azarcón and Alarcón. 
"manifestation of fright" (Rivera 363). There are several other folk-illness concepts related to susto: espanto, susto pasado, colerina and chucaque. "Susto includes all frightening events" (Rivera 363). Espanto refers to the same symptoms as susto but which are more severe (Rivera 363). Some cases of susto and all cases of espanto are thought to be life threatening because it "dislodges the soul (espiritu) from the body" (Rivera 363). The curandera or curandero helps cure the client of susto by helping them to explore and talk about what has caused the fright, in combination with the observance of certain prescribed rituals for treatment (Meizel 153). Susto that is not treated develops to a more severe illness involving soulloss, which is called susto pasado in some regions" (Trotter 173).

This sudden-fright syndrome or susto can be caused by various experiences. The symptoms of espanto are thought to be caused by an encounter with the supernatural, for example, having seen an apparition or ghost (Simmons 61). Simmons also describes the causes as follows: a sudden and unexpected experience such as being startled or attacked by an animal, falling, particularly falling into water, a loud noise or a clap on the back perpetrated by a person whose presence was hitherto unsuspected, and so on, which may or may not involve soul-loss. (57)

The symptoms of fright are numerous, including "wasting away, fever, diarrhea, sleeplessness, loss of will, malaise, and general nervousness" 
(Simmons 61). Rubel notes other symptoms as follows: "restlessness during waking hours, . . . listlessness, loss of appetite, disinterest in costume or personal hygiene, loss of strength and weight, depression and introversion" (278). In addition Kiev mentions other symptoms such as "irritability, asthenia, . . . phobias, reduced libido, retardation, nightmares, trembling, sweating, tachycardia, ... other symptoms of anxiety and depression, and the belief that if the 'fright' reaches the heart, death will occur" (69). In turn, Trotter describes the symptoms as "daytime drowsiness, nighttime insomnia, irritability, jumping at sounds (exaggerated startle reflex), diarrhea" (173).

Rivera mentions that symptoms of susto can also be noticed in children as well, and they are as follows:

crying; whining; sadness; . . . stomach ache; diarrhea; high temperature; vomiting; . . . continuous periods of languor; exhaustion; a loss of interest in all activity; looking pale and thin; shakes or trembles; sore on the body; big round eyes that look sad; and scared of the least little thing. (364) Chucaque and colerina are the other conditions related to susto. The symtoms of Chucaque, which can be caused by embarrassment are said to include "severe aches in the head, stomach, and abdomen, vomiting, diarrhea, fever and chills" (Simmons 61). Colerina can result from anger. Its symptoms include "diarrhea, vomiting and fever" 
(Simmons 61).

In a case of espanto in which soul loss is believed to have occurred, the services of a folk practitioner or curandero are recruited. The folk belief is that "the soul has been captured by magico-spiritual forces which must be placated by the victim and the curer" (812). The folk healers do an invocation and placation of the spirit that is thought to have captured the patient's soul. Cure for susto also involves the use of folk healers to carry out certain prescribed rituals and procedures, using objects, herbs and prayers.

The third folk illness that will be discussed is empacho. The author, who is a nurse, recalls the case of a $30+$ year old male Mexican patient who worked as an agricultural laborer in Oregon who was hospitalized for gastrointestinal symptoms. This patient complained of having empacho and was being treated by a gastroenterologist for his symptoms. The author observed that the patient's family carried out some seemingly benign folk remedies for the patient during the course of the patient's hospitalization: massages and teas. It is certain that health care providers will occasionally encounter Spanish-speaking patients who describe and will attempt to treat folk illnesses as was the author's experience. ${ }^{27}$

${ }^{27}$ This particular episode happened during the time that the author was starting the review of literature for this investigation. 
The folk illness empacho is mainly a disorder characterized by a cluster of gastrointestinal symptoms. Several references cumulatively build a picture of the symptoms, causes, as well as the remedies involved. The symptoms are many and include "lack of appetite, stomachache, diarrhea, and vomiting" (COSSMHO 67); "depression, paleness. . . fever . . . abdominal pains" (Simmons 63); "bloating of the stomach or 'stuffiness' in the stomach or intestines, constipation, indigestion. . . lethargy" (Trotter 175). A variation of causes has been described. Rubel notes that this condition occurs because "a chunk of food clings to the intestinal wall causing sharp pains" (Rubel 799). This is exactly the explanation that the author received from the aforementioned Spanishspeaking patient who was complaining of being empachado. Ingham reiterates this patient's description of the malady saying that it is "caused by a bolus of food that sticks to the wall of the intestine or by some other form of intestinal blockage" (81). Other causes are cited: "it is often suspected that the illness has been caused by the individual having been required to eat against his will" (Rubel 799). Yet another explanation is that it could be due to the ingestion of "green fruit, soft bread, or halfcooked food becoming stuck in the stomach or intestine" (Simmons 63). A wider explanation is that it is caused by: 
Eating improperly cooked foods (eg, [sic] tortillas) or certain foods at the wrong time (eg, [sic] bananas late at night), swallowing chewing gum, swallowing saliva during teething (ie. instead of drooling), and having to eat foods that one does not like. (Trotter 175)

The treatments for this disorder can seem bizzare to health care providers whose main health and illness belief model is biomedical. Trotter describes one of the folk remedies:

The most common treatments for empacho are massages intended to physically dislodge the bolus and ingestion of herbal teas and other substances that are supposed to break up the blockage and flush it out. Diarrhea after treatment is often taken as a sign of successful treatment. (175)

Simmons records the contents of an oral remedy: "a laxative is administered of grated potatoes, salt, lemon, and cooking oil to 'loosen' the object causing the empacho" (Simmons 66). This laxative is given prior to the physical manipulation of the patient by massage. After the massage for empacho, herbal infusions [teas] are given.

Trotter also describes a curious treatment for empacho consisting of massage and physical manipulation:

The second most common treatment is to roll the person onto his or her stomach and grab or pinch the skin in the 
small of the back and pull on it until it 'pops.' The popping sound is taken as an indication that the bolus or blockage has been dislodged. (176).

The third folk treatment involves an even more curious ritual that is further removed from conventional medical therapy: Another common remedy involves rolling an egg (or just the yolk) on the person's stomach while a prayer is recited. The "energy" from this ritual opens the intestines (Trotter 179).

Hot/cold illnesses, susto and empacho and other folk illness symptoms "can be linked to a variety of medical conditions" (Trotter 174) and should be investigated for possible medical intervention. Related biomedically treatable illnesses may range from tuberculosis or gastroenteritis to any of the other aforementioned illnesses documented in Latino health care status reports and statistics.

When directing patient care another major issue to consider is the Latino patient's support system. This includes understanding family dynamics, authority structure, interpersonal relationships and roles. A culturally sensitive health care provider will include the patient's support network to the extent that the patient chooses. Medical-Spanish texts often provide vocabulary for patient's support network but do not give information with regard to interpersonal dynamics according to cultural norms. 
Of course, just as there are differences among individuals there may be differences among family structures, relationships and dynamics. However, the tendency is for close exchange among family members. Luyas writes "The Latino patient typically has close ties to his or her family network" (692). Ryan notes that "Hispanics grow up in a closeknit family network where gregariousness, expressiveness, intimacy, generosity, warmth, affection and mutual support are interrelated and inseperable" (Ryan 33). The family helps to provide "emotional security and sense of belonging" (Hernández 17). If not limited by distance, within the Latino family group there is frequent interaction and visiting (Keefe 68,147 ). Lawson notes "Latino families usually are a source of "social support, tangible aid, information and advice" (78).

Reseach by Hayes-Bautista indicates that the Latino population studied in California, "have high complete nuclear family rates and low non-family rates" (556) and are "more likely than any other group. . . to be a classic nuclear family" (557). Latino patients' support network usually extends beyond "immediate family" to include "extended family and close friends" (Lawson 78). Susan Keefe describes this social network:

Ties beyond the nuclear family are strong and extensive, and reciprocal rights and duties are connected with all relatives including grandparents, aunts, uncles and cousins . . . . 
[C]ompadrazgo or ritual coparenthood associated with choosing godparents, is described as an extension of the kinship system; compadres take on the rights and obligations more characteristic of relatives than friends, and they are included as members of the extended kin network. (144)

Family members help each other, when possible, with material, emotional, and/or spiritual support. The Latino patient may feel a strong sense of duty or responsibility towards family, extended family, or friends ${ }^{28}$ and vice versa.

Some patients choose to confide in their kin when they have medical or emotional problems, while others prefer that family members not "intrude in personal affairs and cause problems" (Keefe 249). Keefe writes that Latino patients "regard familial support for emotional problems as superior to all others" (147). This means that seeking emotional support from close kin is preferable to seeking emotional support from others, such as friends, compadres or comadres; professionals such as therapists, physicians or clergy; neighbors; coworkers; groups etc. (Keefe 148; Guarnaccia 201-202). They may be less likely to seek professional

\footnotetext{
${ }^{28}$ This support network may be so important to the patient that it may affect scheduled patient-provider contacts. For instance, scheduled clinic appointments may be suddenly missed at times due to the sense of responsibility that the patient feels for "attending to the immediate needs of his family or friends" (Hernández 18).
} 
assistance for some concern that is considered a family matter. AntonyTkach writes: "It is stressful for the individual to be seperated from the family group; it is also psychologically difficult for the family to hand over the care of its ill member to a non family health provider" (426).

Guarnaccia notes: "We highlight the value Hispanic families place on taking care of ill family members and the strong sense of responsibility Hispanic families feel for keeping their ill [and convalescent] family members at home" (189). Gonzalez-Swafford writes of the MexicanAmerican family: "It is important not to separate the client from the extended family; therefore, the FNP [Family Nurse Practitioner] may have to include an extended kin group and friends into treatment" (32). When a family functions according to the Latin American ideal, based on the traditional nuclear and extended family, there is a strong support network available to the patient.

In a Latino family it is very likely that family members follow prescribed roles. Reinforced by culture, these roles often are more traditional ones. Hernández writes: "In many respects Latin values are more clearly defined and behavioral patterns are more closely adhered to than is common in the anglo culture" (17). There are exceptions to the rigidness with which these traditional roles are followed. Two prominent exceptions today are the mother being employed outside of the home and the greater participation by fathers in child-care and house-keeping 
activities. An important factor for health care providers to recognize is that patients--especially those who are recent immigrants--may have a harder time adjusting if they are culturally accustomed to having their extended family network accessible to them. If family members did not immigrate together then these patients do not have the extended family network available for support. This contributes to patients' increased stress. They may have to use alternative options for managing affairs of daily living which are non-traditional e.g. child care.

According to the traditional role "[T]he father represents authority within the family. . . All other family members are expected to be respectful of him and to accede to his will or direction" (Hernández 21). The heads of households, who most often are men who are husbands and fathers, have "the ultimate responsibility for making major decisions and controlling the family" (Burk 41). This applies to major decisions about health care matters as well.

This authority arrangement is closely tied to the "concept of machismo or maleness. . . [which entails] using his authority within the family in a just and fair manner" (Hernández 21). Giger and Davidhizar describe this concept as manliness (206). Patricia Caudle comments on some negative aspects associated with machismo as well as the ideal. ${ }^{29}$

${ }^{29}$ Caudel states:

Machismo or male qualities of dominance and 
Health care providers need to acknowledge the familial authority structure when interacting with Spanish-speaking patients. Branch remind providers that in a traditional family arrangement of nuclear and extended family, the cultural norm is for the male head of household the be the "dominant authority" (51). Reinert states one reason why health care providers need to acknowledge this traditional authority structure is because "Mexican-American males find it difficult to tolerate any loss of authority or self-esteem" (30); this has to do with the baffling aspects of machismo built into the culture.

Despite this patriarchal authority structure, in the final decision, family members' opinions are taken into account. This concept "familism" is defined by Phillips as "the tendency for an individual to put the needs of the family above individual needs" (151). The inclusion of the head of household in making family health care decisions is a vital component of gaining cooperation from the patient and the patient's support network. Gonzalez-Swafford notes "[I]nvolvement of the head of

chauvinism have been described by some as a cultural reason why Hispanic men attempt to prove their masculinity through substance abuse, use of prostitutes, and refusal to use condoms. Such choices carry high risk for HIV and AIDS. True cultural machismo, however, demands that men take responsibility for their families and protect them from harm. In this context, machismo becomes pride, dignity, strength, virility, authoritarianism, courage, and honor. (46) 
the family is particularly crucial. By gaining the head of the family's agreement that the client is really ill, the practitioner gains approval for therapy." (32) The head of household can use his authority to help oversee that there is compliance and cooperation by the patient and the patient's support network in accordance to the plan of care agreed upon with the health care provider.

In this traditional family arrangement a woman may refrain from making major medical decisions unless she consults with her husband. Some women will be very adamant about this. To insist that the wife make a major decision without consulting with her husband is a way of quickly cutting off communication (Matta, conference). A study by Clarissa Scott reveals observations about the Puerto Rican population that would be disconcerting to persons of a modern feminist point of view: Many wives and mothers remain close to their homes and neighborhoods and rarely feel comfortable venturing outside these boundaries. Submissive and protected, the Puerto Rican woman in Miami takes direction from her husband (528).

In the traditonal Latin American family arrangement the wife, who possibly is a mother, is assigned the role of caretaker, nurturing and looking after the general well being of the family. As the main caretaker, she takes on the role of assisting with the health care of the ill family 
member which may include administering home remedies or folk remedies. ${ }^{30}$ Other kin or extended family may assist with this also. The main caretaker chooses to seek medical attention when she decides that the illness is "beyond her ability" to treat (Gonzalez-Swafford 29). Lawson notes "Mother directs the affairs of the home including the diagnosing an ill family member and seeking necesary care" (78). Reinert notes:

Medical information is passed down from mother to daughter, and the woman must decide when an illness is beyond her ability to treat. . . [S]he usually discusses the symptoms first with family and friends, then she may utilize folk healers, and finally she may consult a physician. (26) The woman is a key person in the family to recieve health care information, not only because she usually is the main caretaker, but because she is in charge of teaching health care practices to her family. Flaskerud notes "Women play a dominant role in. . . providing the principal source of health education" (438).

In a traditional family arrangement if the wife is the patient, cultural

${ }^{30}$ This has been observed for women of many ethnicities who are in traditional family arrangements:

the task of caring for the sick usually was given to the women, and some became very proficient at healing skills ... [N]ames for these healers varied, depending on the primary ethnic group. .. eg. . . curandera (Bushy 15). 
norms dictate that an explanation of her medical condition and treatment be given to the patient in the presence of her husband, the head-ofhousehold. This is done in consideration of her spouse's concern about her illness and takes into account the patriarchal authority structure. The reverse is also true. If the husband head-of-household is the patient, it is best to share the plan of care in the presence of his spouse and any other supportive kin or extended family the patient wishes to have present. It is necessary for the patient's wife to understand the treatment plan because the women in the family are usually the persons responsible for the care-taking activities (Burk 41).

In the hospital the Latino patient may have many persons visiting who are kin, extended family and/or friends, some of which may choose to stay with the patient to keep him or her company and to help out if possible. It is likely that the Latino patients desire or prefer the participation of close kin or friends and will so indicate if asked. The support and participation of family and extended family can reinforce medical regimens and be a source of aid during a patient's illness or convalescence. Kreisman recommends the "enlistment of family involvement and support" (83). As in most clinical teaching situations, it is better to have more than one family member present when explaining and teaching a prescribed self-care task, e.g. blood sugar testing, dressing changes etc. If the skill can be observed by several persons who will be 
involved in the patient's care, the skill can be discussed and returndemonstrated by not only the patient but also by persons of his or her support network. They can monitor each other and assist with the performance of the hands-on skills taught them. By reminding each other about the details of what needs to be done, they reinforce what has been taught them.

The presence of the support group, which is positive for the patient, can be managed in the hospital environment to keep disturbances to a minimum. There may be a cultural incongruence between patients wanting their social support groups present and the hospital personnel's need to keep the hospital enviroment low key. Rella Adams describes a common instance affecting hospital in-patients, especially in intensive care situations: "With hospitals' restrictive visiting hours and numbers, the MA [Mexican-Americans] are prevented from using their support system, which usually extends beyond their immediate family" (111). The expectation is that visitors remain low key while in the hospital environment. Hospital personnel may feel obligated to enforce limitation of visitors if the presence of the patient's support group disturbs the theraputic enviroment. Instances which disturb the hospital environment include increased noise, crowding in the patient's room and other areas, children who are excessively loud and whose behavior is not well controlled by their parents, etc. Some of the disturbances are innate to 
having persons congregate. Other disturbances can be controlled by communication between hospital personnel and the visiting parties.

Allowance of the presence of the patient's support group can be made and encouraged if it is monitored and tactfully managed by the provider.

The health care provider needs to assess the Latino patient's support system as it is integral to the care and well being of the patient. When this arrangement does not exist for some reason, the Latino patient will feel the absence of these close ties which are cultural norm. Keefe addresses this saying:

[W] hen a family support system is absent, not "wellintegrated" or one in which there is "malfunction" . .

[This] is probably far more distressing [for the Latino patient] than for Anglo Americans in similar circumstances. . . [with] "intensified isolation or stress." (151)

As for any patient, alternate sources of support need to be arranged if there is not family or extended family available to assist with the patient's care.

The atmosphere of close exchange with family members has an impact on the Latino patient's communication style outside of the family. This brings up another cultural health-related issue. Latino patients prefer and expect a certain communication style to be used in clinical interactions. They are likely to judge the medical attention received by 
the quality of the technical skill of the health care provider but also by the interpersonal interaction with the health care provider. The communication style valued and expected by a client is based on diplomacy (Burk 40), tactfulness (Burk 40) and respect which are based in common sense. The concept of respeto (respect) is defined by Maduro as a sense of "decency, or a common humanness" in people's interaction with one another (874). Burk comments that respect "dictates appropriate deferential behavior towards others on the basis of age, sex, social position, economic status, and position of authority" (40). According to $M$. Leininger, "respect for those in authority" is a cultural value that was considered to be closely associated to "quality of life" by persons of the Mexican American population in the study (27).

The cultural ideal is for respect to be demonstrated in family relationships as well as in other interactions. For instance, in family relationships "respect and obedience to elders" is expected (Branch 51). Lapierre speaks of a hierarchy in various relationships "Elders expect respect from younger individuals, adults from children, men from women, teachers from students, employers from employees" (38). Antony-Tkach describes that respect "makes use of much diplomacy, tact, concern, and respect for another's feelings. Direct argument or contradiction is considered rude or disrespectful" (427).

The concept of respect is relevant to [the] provider-client 
relationship as well. In the health care provider-patient relationship the expectation of respect is reciprocal:

Mexican-American clients respect health care providers as authority figures. In return, they expect to be treated with respect by providers. Respeto is demonstrated by using formal, rather than informal, Spanish language and by listening attentively to clients and providing information in a courteous manner. (Burk 40)

The establishment of respect requires active demonstrative effort on the part of health care providers as well as the patients. In the medical setting it is better not to assume that this respectful understanding between provider and client is present (Siantz 38; Lapierre 39).

Adequate patient-provider rapport is important to establishing trust in the theraputic interaction and treatment. If patients feel that they are being listened to and that their concerns are being addressed, this will help to foster patient trust and cooperation which, in turn contributes to better compliance with the medical treatments prescribed and improved theraputic results. A Latino patient will talk about his or her medical or emotional problems "[i]f there is confianza, which is understanding, confidentiality, and trustworthiness" (Keefe 149). A culturally sensitive health care provider who in addition speaks Spanish can inspire a sense of confianza in patients. 
Most non-Latino health care personnel are "taught to value openness, frankness, and directnessl . . . [They are] more likely to express [themselves] simply, briefly, and frequently bluntly" (Hernández 18). Time constraints of the clinical situation can accentuate the use of this style of communication. This type of communication may be in cultural contrast to the interaction that the Latino patient expects from health care providers. Due to marked cultural contrast "the Anglo often seems to be cold, distant, and lacking in sensitivities" (Hernández 19). A clinician who is direct, blunt and confrontational may foster distrust and not gain the patient's respect and cooperation.

Another aspect of communication style that is favored by Latino patients reflects "personalism. . . and informality in institutional and interpersonal relations. . . . [Once a person is considered] 'part of the family, the communication can move more rapidly" (Ryan 34). A sense of personalism in the professional patient-provider interaction will help to develop trust; however, "there will always be some distance or 'line of authority' that is difficult to cross" (Ryan 36). Gestures in accord with the concept of personalism include politely greeting the patient with a handshake or hug if it is of an established and more intimate providerpatient relationship; asking about the well-being of the patient's family or about any significant and informal detail of the person's life deemed appropriate. Greeting only the patient and not greeting his or her support 
network defies the cultural norm of personalism (Matta, conference). Although there may be friendly conversation of a personal nature beginning the provider-patient interaction, the person providing the medical attention will be shown repect by the patient, thus maintaining the fine line of professional distance.

Health care providers are expected to have the required technical skills as well as to show sensitivity and extend courtesy towards the client (Hernández 19). The concept of courtesy can add an element of misunderstanding to the patient-provider interaction. When the health care provider speaks or gives instructions to the patient, the Latino patient may follow what is being said with gestures or utterances such as a nodding of the head up and down or saying something to the effect of "sí" or some other utterance. These gestures or utterances often are interpreted by health care providers to suggest that the patient is understanding and is in agreement with what is being said or explained.

The misunderstanding lies in the fact that the patient may be doing this automatically, out of respect, not wanting to interrupt or show difference of opinion. In reality the patient may not understand what is being explained, may have questions, would like to comment on what the provider has said, or may even disagree with what is said, but will be reluctant to express this (Matta, conference). Hernández notes that the patient may "not reveal his genuine opinion openly unless he knows the 
other well and unless he can take time to tactfully differ" (19). Thus these patient gestures lead the health care provider to "falsely assume that basic agreement has been reached" (Hernández 19).

The culturally aware provider will recognize the possibility of differences in communication styles and will be able to tactfully elicit the patient's comments, quesitons or opionions. The point is that the provider should verify that the patient has an accurate understanding of or agrees with the instruction or information given. One technique is for providers to ask patients to repeat back in their own words the information and instructions given (Matta, conference). Clarification and written information should be given to the extent to which it is needed. This is applicable to all patients, not only the Latino patients, because often the anxiety of the situation may decrease a patient's ability to comprehend and retain information.

In communication, body language is as important as verbalizations. Communicating with a patient in Spanish has as much to do with the manner in which the message is conveyed, as with the accuracy of it. Respect is displayed through both attitudes and actions taken, including non-verbal behavior. Burk recommends that "the primary care provider's nonverbal communication, such as body language, be consistent with his or her verbal communication (40). Saintz notes salient characteristics of Latino patients' communication style "[Communication style] may be 
more animated; their [the patient's] need for physical closeness with less personal body space required; eye contact. . . may be expected of the nurse but not necessarily of the client" (38). Lapierre writes about eye contact: "Traditionally, Hispanics have been taught to avoid eye contact with authority figures. . . as a sign of respect. Such behavior should not be misinterpreted as disinterest in communication" (38). Another point of view is that there is avoidance of eye contact "only from working class people, middle class doesn't follow this pattern" (Cabello, Thesis Commitee). The goal of the provider-client interaction is to establish and maintain clear communication on several levels during the therapeutic interactions: verbal and non-verbal. The aforementioned are details that are not conveyed to the language student in the status quo Spanish-forhealth care-providers books on the market. They can be understood on a deeper level by receiving both transcultural information and experiential learning in the clinical setting.

Locus of control issues will come up at times when working with Latino patients. Locus of control is the "perception" people have of "control over their lives" (McAllister 1449). Patients of the Spanishspeaking community hold differing perceptions of the control they have of their lives and health. Locus of control--internal or external--affects patients' health care attitudes and behaviors. Persons with an internal locus of control believe that "outcomes are contingent on one's own 
intentions, choices and behavior" (Mirowsky 2). Health care providers will find that Latino patients with internal locus of control are more apt to take on active responsibility for their own health care. This is because they are "more likely to believe that health is the result of their own behavior" (Mcallister 1450). Patients' accountability for their own health is something that health care professionals encourage and expect of patients. In other instances health care providers will find that despite their best efforts to encourage patient participation, some Latino patients choose not to take responsibility for their own health care and do not follow through with medical recommendations. Since health care providers are trained to expect and encourage patients to actively participate in their own health care, this is a difficult and, at times frustrating, situation for health care providers to encounter.

If patients have an external locus of control and health care providers have an internal locus of control and expect this of the patients they deal with, a cultural clash of perspectives will become apparent. Rather than carrying out actions beneficial to their health, some Latino patients with an external locus of control have a fatalistic perspective and choose passive acceptance. McAllister writes that patients with an external locus of control "will be less likely to comply with officially recommended health programmes [sic]" (1449). Patients with an external locus of control believe that "outcomes of situations are controlled by 
forces external to one's self, such as powerful others, luck, fate, or chance. . . he or she is more or less at the mercy of the environment" (Mirowsky 2). This belief predisposes patients to not want to take responsibility for their health, since they perceive that it will not make a difference.

Burk writes:

The fatalistic view demonstrated by many Mexican Americans reflects an external locus of control. They believe that life and health are controlled by divine will, fate, and the environment. Consequently, the individual perceives little personal ability or responsibility for success or failure in matters of health and illness. (43)

The attitude that one cannot change situations in one's life can lead to "feelings of powerlessness" and "passive acceptance" (Adams 110). There are expressions in the Spanish language that reflect a fatalistic point of view, such as "Que sea lo que Dios quiera" (Adams 110), "Si Dios quiere (If God wishes)" or "Que Dios nos bendiga (May God bless us)" (Guarnaccia 187). These sayings reflect a resignation to and passive acceptance of what is to happen. Calvillo writes about the meaning behind these kinds of expressions:

[I]n many Hispanic cultures the value of suffering and the concept of fatalism are accepted beliefs with religious 
undertones. Many Hispanic persons believe that life has many difficulties which must be accepted without complaints .... If a person is ill, that person bears the illness with dignity and courage. Many are able to progress through an illness or through a recovery period with this attitude because "everything is in the hands of God" (Calvillo 17).

Guarnaccia comments about the religious undertones of such expressions: "These expectations reflect Hispanic families' strong religious beliefs and, at the same time, the hopes and frustrations they experience in dealing with illness" (287). Another saying--"The doctor's power to heal comes through God and God imbues medicines with the strength to overcome illness" (Guarnaccia 206)--reveals a favorable attitude towards the use of medical services. Examining the health care beliefs of Latino patients can reveal a curious combination of religious belief, fatalism and passive acceptance.

Garza examines fatalism in the Latino population and states that this is not a characteristic unique to Latinos: "fatalism is a function of socioeconomic background" or "socioeconomic status" (133). Mirowsky has a similar opinion: "people from lower-income families are more likely to believe in external control than are people from higher income families" (9). Mirowsky goes on to explain: "Failure in the face of effort leads to belief in external control which can increase passivity and giving up. . . . 
Success leads to belief in internal control, characterized by an active problem solving approach to life" (2). A fatalism reinforced by socioeconomic status can reflect in health care attitudes of patients. Another point of view is that Latino patients show strong internal locus of control. This is demonstrated through self control:

[T] he concept of self-control is an important element in the Mexican culture. Self-control (controlarse) includes: 1) the ability to withstand stress in times of adversity (aguantarse); 2) a passive resignation in which the person accepts his or her fate (resignarse); or 3 ) a more active cognitive coping which means working through a problem (sobreponerse). (Calvillo 19)

Garza says that the Latino population demonstrates successful control of their environment by retaining their language and cultural practices: "family-centered orientation. . . and the perennial resistance to give up their culture and heritage. . suggest belief in internal locus of control" (Garza 134). Garza goes on to state that "family-centered environments could contribute to a greater perception of internal control" (133). Mirowsky talks about the role of family support: "family and extended family help to provide a sense of 'security'" (3). A strong family support system helps to enhance an individual's coping ability, helping family members to work through their problems or sobreponerse. Garza 
points out another indication of internal locus of control and self-control among the Latino population: they are "usually polite and respectful towards others even when it is not an expression of true feelings" (Garza 135).

Information in journal articles state that there are elements of both internal and external locus of control among Spanish-speaking patients. Each patient will have his or her own point of view, which will be reflected in their health care behavior. Some patients are willing to accept medical recommendations and carry out positive actions for taking care of their health or the health care of family members. There are other patients who will resist involvement. Professionals can gain some insight into the patients' cooperative and uncooperative behavior if cultural bases are taken into consideration, part of which includes understanding the patient's locus of control. 


\section{Conclusion}

Vast resources are spent on providing health care to the Latino population in the U.S. However, because of both language and cultural barriers, medical care services are not efficiently provided to the Spanishspeaking client population. This inefficiency of services wastes both money and the limited time of qualified medical personnel. Because current medical problems are not resolved, they will result in higher medical and social costs in the future.

If not solved now, inefficient use of health care resources will continue to increase. The U.S. Latino population is growing far faster than the population as a whole, and an ever higher percentage of the Latino population is coming into contact with the health care system as they participate in the mainstream economy, thus gaining access to medical care.

One obvious solution is to attract more of the Latino population to the health care professions. Recruitment efforts have been controversial and have met with very limited success. The percentage of Latino medical, nursing and allied health providers is still a small fraction of the percentage of patients who are Latino in the U.S.

Therefore, we must effectively train medical personnel who do not already have the cultural and language background necessary to provide good and efficient care to Latinos. Health care professionals who can 
take patients' cultural health care practices into consideration will have much better insight into patients' cooperative and uncooperative behavior. They will be better able to address the health care needs of this particular patient population and formulate culturally relevant interventions. The benefits and savings as a result of Spanish language and cultural training will far outweigh the costs of providing the instruction. Based on a review of several readily accessible texts for teaching Spanish to health care providers and an investigation of the availability of classes for teaching Spanish to health care providers in Portland, Oregon, it is clear that resources currently available for providing such language and culture training are sorely lacking in both quantity and quality. Instruction on cultural issues relevant to Latino health care needs to be incorporated into specialized Medical Spanish language training in order for the instruction to be more effective than what is available today. Not only do we need more classes for teaching Spanish language and culture to health care professionals at more Universities, and in-house or inservice programs in the clinical environments, but such training needs to be made more practical and pertinent to the real world clinical settings and situations in which providers and clients interact. The texts and curricula material must also facilitate a practical learning experience that realistically simulates the work site experiences. Language instruction must present and stimulate discussion about culturally specific Latino health-related 
issues. A reformulation of texts and curriculum used for medical-Spanish language instruction would include instruction on the basics of cultural assessment; dialogues or conversation models that have built into them health-related cultural issues for discussion; case studies that raise cultural issues for discussion and which can be used for conversation exercises; subgroup specific cultural information; specifics about linguistic regionalisms among subgroups; ample bibliography for further investigation of issues if desired, etc. Some of the pedagogical exercises which are commonly found in the Medical Spanish texts already published could be reworked so as to incorporate this type of focus.

In this study the author has addressed only a few of the many cultural health-related issues of which health care providers need to be aware when interacting with Latino patients. In order to end the waste of human and economic resources, texts must be developed and more pertinent curricula must be implemented. Reaching medical providers with such training would help facilitate the provision of more effective and culturally relevant medical care to the Spanish-speaking patient population. 
Appendix

Texts examined by the author

Artschwagner, Margarita. Southwestern Medical Dictionary. University of Arizona Press: Tucson, 1977.

Bongiovanni, Gail. Medical Spanish. McGraw-Hill: New York, 1991.

Cordasco, Francesco, and Pablo Alvarez. Useful Spanish for Medical and Hospital Personnel with a Bibliography on Hispanic Peoples in the United States. Blaine-Ethridge Books: Detroit, 1977.

Di Lorenzo-Kearon, Maria, and Thomas Kearon. Medical Spanish: $A$ Conversational Approach. Holt, Rinehart and Winston: Fort Worth, 1981.

Geissler, Elaine. Pocket Guide: Cultural Assessment. Mosby: St. Louis, 1994.

Harvey, William. Spanish for Health Care Professionals. Barron's: New York, 1994.

Howell, Grace, and Jesús Pérez y Sabido. Spanish English Handbook Compendio en Inglés y Español. Medical Economics Company: Oradell, 1977.

Kantrowitz, Martin, Antonio Mondragón, and William Coleman. ¿Qué Pasó: A English-Spanish Guide for Medical Personnel. University of New Mexico Press, 1993.

Keeland, Brent, and Lou Jordan. Communimed: Multilingual Patient 
Assessment Manual. Mosby Lifeline: St Louis, 1994.

Kite, Ralph. Destinos: Spanish for the Medical Professions. McGrawHill: New York, 1993.

Lebredo, Jarvis. Spanish for Medical Personnel. D.C. Heath and Company: Lexington, 1996.

Loeb, Stanley, Ed. English and Spanish: Medical Words and Phrases. Springhouse: 1994.

Meizel, Janet, E. Spanish for Medical Personnel. Skidmore-Roth: Davis, 1993.

Pérez-Sabido, Jesús. Spanish English Handbook for Medical Professionals Compendio en Inglés y Español Para Profesionales de la Medicina. Practice Management Information Corporation: Los Angeles, 1994.

Rajikovic, Ana. Manual for Painless Medical Spanish: A Self-Teaching Course. University of Texas Press: Austin, 1992.

Rios, Joanne. Converse en español: español para personal médico. Rios Associates: Tucson, 1988.

Rogers, Glenn. English-Spanish, Spanish-Engish Medical Dictionary. McGraw-Hill: New York, 1992.

Schmitt, Conrad J., and Protase E. Woodford. Medicina y Servicios Médicos. McGraw-Hill: New York, 1992.

Speedy Spanish for Medical Personnel. Baja Books: Santa Barbara, 
1988.

Speedy Spanish for Nursing Personnel. Baja Books: Santa Barbara, 1988. 
Works Consulted

Adams, Rella, et al. "Cultural Considerations: Developing A Nursing Care Delivery System For A Hispanic Community." Nursing Clinics of North America 27.1 (1992): 107-116.

Alinger, Rita L. "Folk Beliefs About High Blood Pressure in Hispanic Immigrants." Western Journal of Nursing Research 10.5 (1988): 629-637.

--.. "Patients' Explanation of Rheumatoid Arthritis."

Western Journal of Nursing Research 15.3 (1993): 341-351.

Alvarez, Julia. "Does color matter to us?" Latina Fall 1996: 50-54.

Andrews, Margaret M. "President's Message." Journal of Transcultural

Nursing 5.2 (1994): 47.

Anthony-Tkach, Catherine. "Care of the Mexican-American Patient."

Nursing \& Health Care 2.8 (1981): 424-432.

Asch Steven. "Does Fear of Immigration Authorities Deter Tuberculosis Patients From Seeking Care?" Western Journal of Medicine 161.4 (1994): 373-376.

Bellack, Janis P. Nursing Assessment A Multidemensional Approach.

Monterey: Wadworth Health Sciences Division, 1984.

Berkanovic, Emil. "The Effect of Inadequate Language Translation On Hispanic's Responses to Health Surveys." American Journal of 
Public Health 70.12 (1980): 1273-1276.

Bern, Caryn., et al. "Diarrheal deaths in children living in New Mexico: Toward a strategy of preventive interventions." The Journal of Pediatrics 122.6 (1993): 920-921.

Bernal Henrietta. "A Model for Delivering Culture-Relevant Care in the Community." Public Health Nursing 10.4 (1993): 228-232.

---., et al. "Rewards and Frustrations of Working with an Ethnic Minority Population: An Hispanic Unit Experience." Home Healthcare Nurse 8.3 (1990): 19-23.

Berry, Anita. Rev. of Transcultural Nursing: Concepts, Theories, Research, and Practices, by M. Leininger. Journal of Transcultural Nursing 7.2 (1996): 41-42.

Branch, Marie. Providing Safe Nursing Care for Ethnic People of Color. New York: Appleton-Century-Crofts, 1976.

Bray, Martha L., et al. "A Primary Health Care Approach Using Hispanic Outreach Workers as Nurse Extenders." Public Health Nursing 11.1 (1994): 7-11.

Burk, Martha E., et al. "Cultural beliefs and health behaviors of pregnant Mexican-American women: Implications for primary care." Advances in Nursing Science 17.4 (1995): $37-52$.

Bushy, Angeline. "Cultural considerations for primary health care: Where do self-care and folk medicine fit?" Holistic Nursing Practice 6.3 
(1992): 10-18.

Calvillo, Evelyn R. and Jacquelyn H. Flaskerud. "Review of Literature on Culture and Pain of Adults with Focus on Mexican-Americans." 2.2 (1991): 16-23.

Capers, Cynthia Flynn. "Teaching cultural content: A nursing education imperative." Holistic Nursing Practice 6.3 (1992): 19-28.

Carpio, Barbara A., and Basanti Majumdar. "Experiential Learning: An approach to transcultural education for nursing." Journal of Transcultural Nursing 4.2 (1993): 4-11.

---. "Putting Culture into Curricula." Canadian Nurse 87.7 (1991): 32 33.

Casewell, Evans A. "Immigrants and Health Care: Mounting Problems." Annals of Internal Medicine 122.4 (1995): 309-310.

Caudle, Patricia. "Providing culturally sensitive health care to Hispanic clients." Nurse Practitioner: American Journal of Primary Health Care 18.12 (1993): 40, 43-44, 46.

Chandler, Charles R. "Traditionalism in a Modern Setting: A Comparison of Anglo- and Mexican-American Value Orientations." Human Organization 38.2 (1979): 153-159.

Chatman, Joy. "AT\&T Language Line Services." Letter to the author. 12 September 1996.

Chesney, Alan P. "Mexican-American Folk Medicine: Implications for the 
Family Physician." The Journal of Family Practice 11.4 (1980): 567-574.

Clabots, Renee B. "The Multilingual Videotape Project: Community Involvement in a Unique Health Education Program." Public Health Reports 107.1 (1992): 75-80.

Clark, Margaret M. "Cross-cultural Medicine: Cultural Context of Medical Practice." The Western Journal of Medicine 139.6 (1983): 806810.

Council on Scientific Affairs. "Hispanic Health in the United States." Journal of the American Medical Association 265.2 (1991): 248252.

Cravener, Patricia. "Establishing Theraputic Alliance Across Cultural Barriers." Journal of Psychosocial Nursing 30.12 (1992): 10-14, 37-38.

Creighton, Margo Neal, et al. Nursing Care Planning Guides for Psychiatric and Mental Health Care. Baltimore: Williams \& Wilkins, 1985.

Cuellar, Israel. "Cultural Considerations and Rehabilitation of Disabled Mexican Americans." Journal of Rehabilitation 54.3 (1988): 3541.

Currier, Richard L. "The Hot-Cold Syndrome and Symbolic Balance in Mexican and Spanish-American Folk Medicine." Ethnology 5.2 
(1966): 251-263.

Dawson, William D. "School District and School Personnel Concerned About the Implementation of Proposition 187." Acting State Superintendent of Public Instruction memo to County and District Superintendents of Public Instruction. California Dept. of Education. Sacramento, California. 9 November 1994.

Dehn, Marlene A. "Vitamin C, Chicken Soup, and Amulets: Students view self-care practices." Nurse Educator 15.4 (1990):12-15.

DeSantis, Lydia. "Developing Faculty Expertise in Culturally Focused Care and Research." Journal of Professional Nursing 7.5 (1991): 300309.

Díaz-Duque, Ozzie F. "Advice from An Interpreter." American Journal of Nursing 82.9 (1982): $1380-1382$.

Douglas, M. "President's Message: culturally competent nursing care." Journal of Transcultural Nursing 6.2 (1995): 43-44.

Edgerton, Robert B. "Curanderismo in the Metropolis." American Journal of Psychotherapy 24.1 (1970): 125-134.

Eliason, Michele J. "Ethics and transcultural nursing care" Nursing Outlook 41.5 (1993): 225-228.

Engel, Samuel., et al. "Diabetes Care Needs of Hispanic Patients Treated at Inner-City Neighborhood Clinics in New York City." The Diabetic Educator 21.2 (1995): 124-128. 
Fahy, Ellen T. "Transculturalism: Still Winking and Speaking in Code." Nursing \& Health Care 15.5 (1994): 227.

Ferketich, Sandra, et al. "Focus on Psychometrics: Development and Administration of a Survey Instrument for Cross-Cultural Research." Research in Nursing \& Health 16.3 (1993): 227-230.

Finkler, Kaja. Spiritualist Healers in Mexico. South Hadley: Bergin \& Garvey Publishers, 1985.

Finn, Julianna M. "A Transcultural Nurse's Adventures In Costa Rica:

Using Leininger's Sunrise Model For Transcultural Nursing

Discoveries." Journal of Transcultural Nursing 4.2 (1993): 19-23.

Flaskerud, Jacquelyn $\mathrm{H}$. "Beliefs about AIDS, Health, and Illness among Low-Income Latina Women." Research in Nursing \& Health 14.6 (1991): $431-438$.

Ford, Guthrie J. "Differences between Mexican-American and White Children in Interpersonal Distance and Social Touching." Perceptual and Motor Skills 45.3 (1977): 779-785.

Ford, Karen Cowan. Las Yerbas de la Gente: A Study of HispanoAmerican Medicinal Plants. Ann Arbor: University of Michigan, 1975.

Forrest, Katherine A., et al. "Exploring Norms and Beliefs Related to AIDS Prevention Among California Hispanic Men." Family Planning Perspectives 25.3 (1993): 111-117. 
Furino, Antonio. "Health Status Among Hispanics: Major Themes and New Priorities." Journal of the American Medical Association 265.2 (1991): 255-257.

Germain, Carol P. "Cultural care: A bridge between sickness, illness, and disease." Holistic Nursing Practice 6.3 (1992): 1-9.

Giger, Joyce and Ruth Davidhizar. Transcultural Nursing: Assessment and Intervention. St. Louis: Mosby, 1995.

Gillin, John. "Magical Fright." Psychiatry 11.0 (1948): 387-400. Gonzalez, Cristine. "Healer treats mind, body, spirit." Oregonian 8 Oct. 1996: B1, B3.

Gonzalez-Swafford, Maria J., and Mary Grace Gutierrez. "Ethno-Medical Beliefs and Practices of Mexican-Americans." Nurse Practitioner 8.10 (1983): 29-34.

Grasska, Merry Ann and Teresa McFarland. "Overcoming the Language Barrier: Problems and Solutions." American Journal of Nursing 82.9 (1982): 1376-1379.

Guarnaccia, Peter J., et al. "Si Dios Quiere: Hispanic Families' Experiences of Caring For a Seriouly Mentally III Family Member." Culture, Medicine and Psychiatry 16.2 (1992): 187-215.

Guendelman, Sylvia. "Fetal Deaths in Mexican-American, Black, and White Non-Hispanic Women Seeking Government-Funded Prenatal Care." Journal of Community Health 19.5 (1994): 319-330. 
Haffner, Linda. "Cross-Cultural Medicine, A Decade Later: Translation Is Not Enough, Interpreting in a Medical Setting." The Western Journal of Medicine 157.3 (1992): 255-259.

Hartog, Joseph., and Elizabeth Ann Hartog. "Cultural Aspects of Health and IIIness Behavior in Hospitals." The Western Journal of Medicine 139.6 (1983): 910-916.

Harwood, Alan. "The Hot-Cold Theory of Disease: Implications for treatment of Puerto Rican Patients." Journal of the American Medical Association 216.7 (1971): 1153-1158.

Hayes-Bautista, David E., et al. "Latino Health in California, 1985-1990: Implications for Family Practice." Family Medicine 26.9 (1994): $556-562$.

--.. "Latino Terminology: Conceptual Bases for Standardized Terminology." American Journal of Public Health 77.1 (1987): 6168.

---. "Latino Terminology: Conceptual Bases for Standardized Terminology." American Journal of Public Health 77.1 (1987): 6168.

Helman, Cecil G. Culture, Health and IIIness: An introduction for health professionals. Boston: Wright, 1990.

Hernández, Carrol A. Chicanos: Social and Psychological Perspectives.

St. Louis: C.V. Mosby, 1976. 
Herrera, Carlos R., et al. "Mortality Among Hispanics." Journal of the American Medical Association 271.16 (1994): 1237.

Higginbotham, John C. "Utilization of Curanderos by Mexican Americans: Prevalence and Predictors Findings from HHANES [Hispanic Health and Nutrition Examination Survey] 1982-84." AJPH 80 Supplement (1990): 32-35.

Holland, William R. and Roland G. Tharp. "Highland Maya

Psychotherapy." American Anthropologist 66.1 (1964): 41-52.

Huttlinger, Kathleen W. "The peyote way: implications for culture care theory." Journal of Transcultural Nursing 5.2 (1994): 5-11. Ingham, John M. "On Mexican Folk Medicine." American Anthropologist 72.0 (1970): $77-87$. Institute for Health Professionals: Continuing Education for Nurses Fall 1996. Portland: Portland Community College, 1996. Irwin Press. "The Urban Curandero." American Anthropologist 73.3 (1971): 741-756.

It May Be For You: The Oregon Health Plan, A Medicaid Program.

Portland: Oregon Health Plan, 1996.

Juarbe, Teresa C. "Access to Health Care for Hispanic Women: A Primary Health Care Perspective." Nursing Outlook 43.1 (1995): 23-28.

Karno, Marvin and Robert B. Edgerton. "Perception of Mental Illness in a 
Mexican-American Community." Archives of General Psychiatry 20.0 (1969): 233-238.

Kaufert, Joseph M., and William W. Koolage. "Role Conflict Among 'Culture Brokers': The Experience of Native Canadian Medical Interpeters." Social Science \& Medicine 18.3 (1984): 283-286.

Keefe, Susan. "Real and Ideal Extended Familism Among Mexican Americans and Anglo Americans: On the Meaning of "Close" Family Ties." Human Organization: Journal of the Society for Applied Anthropology 43.1 (1984): 65-70.

--.. "The Mexican-American Extended Family As An Emotional Support System." Human Organization: Journal of the Society for Applied Anthropology 38.2 (1979): 144-152.

Kiev, Ari. Transcultural Psychiatry. New York: The Free Press, 1972.

Kirkman-Liff, Bradford and Delfi Mondragón. "Language of Interview:

Relevance for Research of Southwest Hispanics." American Journal of Public Health 81.11 (1991): 1399-1404.

Kraut, Alan M. "Healers and Strangers: Immigrant Attitudes Toward the Physican in America-A Relationship in Historical Perspective." Journal of the American Medical Association 263.13 (1990): $1807-1811$.

Kreisman, Jerold J. "The Curandero's Apprentice: A Theraputic Integration of Folk and Medical Healing." American Journal of 
Psychiatry 132.1 (1975): 81-83.

Lapierre, Earnest D., and Joan Padgett. "Answers Professionally

Speaking: How can we become more aware of culturally specific body language and use this awareness theraputically?" Journal of Psychosocial Nursing 29.11 (1991): 39-41.

Lawson, Lauren V. "Culturally Sensitive Support." American Journal of Maternal Child Nursing 15.2 (1990): 76-79.

Leininger, Madeleine. "Becoming aware of types of health practitioners and cultural imposition." Journal of Transcultural Nursing 2.2 (1991): 32-39.

---. "Cultural care: an essential goal for nursing and health care." AANNT Journal: American Association of Nephrology Nurses \& Technicians 10.5 (1983): 11-17.

---. "Culturally competent care: visible and invisible." Journal of Transcultural Nursing 6.1 (1994): 23-25. . "Culture Care of Mexican Americans." Transcultural Nursing:

Concepts, Theories, Research \& Practice. New York: McGraw-Hill, 1995. 365-382.

..-. "Leininger's Acculturation Health Care Assessment Tool For Cultural Patterns In Traditional and Non-Traditional Lifeways." Journal of Transcultural Nursing 2.2 (1991): 40-43.

---. "Quality of Life from a Transcultural Nursing Perspective." Nursing 
Science Quarterly 7.1 (1994): 22-28.

---. "Self-Care Ideology and Cultural Incongruities: Some Critical Issues." Journal of Transcultural Nursing 4.1 (1992): 2-4.

-.-. "Teaching Transcultural Nursing in Undergraduate and Graduate Programs." Journal of Transcultural Nursing 6.2 (1995): 10-21.

--.. "Transcultural Nursing Education: A Worldwide Imperative." Nursing \& Health Care 15.5 (1994): 254-257.

Lieu, Tracy A., et al. "Race, Ethnicity, and Access to Ambulatory Care among US Adolescents." American Journal of Public Health 83.7 (1993): 960-965.

Lopez, Laureen M. and Renoit R. Masse. "Income, Body Fatness and Fat Patterns in Hispanic Women From The Hispanic Health And Nutrition Examination Survey." Health Care for Women International 14.0 (1993): 117-128.

Luckmann, Joan, and Karen Creason Sorensen. Medical-Surgical Nursing: A Psychophysiologic Approach. Philadelphia: W. B. Saunders, 1980.

Luyas, Gloria T. "An Explanatory Model of Diabetes." Western Journal of Nursing Research 13.6 (1991): 680-695.

Maduro, Renaldo. "Curanderismo and Latino Views of Disease and Curing." The Western Journal of Medicine 139.6 (1983): 868874. 
Manson, Aaron. "Language Concordance as a Determinant of Patient Compliance and Emergency Room Use in Patients with Asthma." Medical Care 26.12 (1988): 1119-1128.

Marcos, Luis R. "Understanding Ethnicity in Psychotherapy with Hispanic Patients." The American Journal of Psychoanalysis 48.1 (1988): $35-41$.

Mardiros, Marilyn. "A view toward hospitalization: the Mexican American experience." Journal of Advanced Nursing 9.5 (1984): 469-478.

Marin, Gerardo, et al. "The Development of a Relevant and Comprehensive Research Agenda to Improve Hispanic Health." Public Health Reports 108.5 (1993): 546-550.

Marks, Gary., et al. "Health Risk Behaviors of Hispanics in the United States: Findings from HHANES, 1982-84." American Journal of Public Health 80 Supplement (1990): 20-26.

Martaus, Therese M. "The Health-seeking Process of Mexican-American Migrant Farmworkers." Home Healthcare Nurse 4.5 (1986): 32 37.

McAllister, Gillian and Morag Farquhar. "Health beliefs: a cultural division?" Journal of Advanced Nursing 17.12 (1992): 14471454.

Meister, Joel S., et al. "Using Lay Health Workers: Case Study of a 
Commumity-Based Prenatal Intervention." Journal of Community Health 17.1 (1992): 37-51.

Mikhail, Blanche I. "Hispanic Mothers' Beliefs and Practices Regarding Selected Children's Health Problems." Western Journal of Nursing Research 16.6 (1994): 623-638.

Mirowsky, John and Catherine E. Ross. "Mexican Culture and its Emotional Contraditions." Journal of Health and Social Behavior 25.1 (1984): $2-11$.

Mobed, Ketty. "Cross-cultural Medicine A Decade Later: Occupational Health Problems Among Migrant and Seasonal Farm Workers." The Western Journal of Medicine 157.3 (1992): 367-373.

Moore, Joan W. Mexican Americans. Englewood Cliffs: Prentice-Hall, 1976.

Moore, Patricia and Joseph T. Hepworth. "Use of Perinatal and Infant Health Services by Mexican-American Medicaid Enrollees." Journal of the American Medical Association 272.4 (1994): 297-304.

Moy, Ernest and Barbara A. Bartman. "Physician Race and Care of Minority and Medically Indigent Patients." Journal of the American Medical Association 273.19 (1995): 1515-1520.

National Coalition of Hispanic Health and Human Services Organizations 1996 Catalog: Consumer Materials, Tranining Materia/s, Professional Resources, Policy and Research Materials, Hot Line 
Information. Washington D.C.: COSSMHO, 1996.

Ness, Robert C., and Ronald M. Wintrob. "Folk Healing: A Description and Synthesis." American Journal of Psychiatry 138.11 (1981): 1477-1481.

Neves Arruda, Eloita., et al. "Comfort: Immigrant Hispanic cancer patients' views." Cancer Nursing 15.6 (1992): 387-394.

Nkongho, Ngozi 0 . "Teaching health professionals transcultural concepts." Holistic Nursing Practice 6.3 (1992): 29-33.

Niederhauser, Victoria P. "Health Care of Immigrant Children: Incorporating Culture Into Practice." Pediatric Nursing 15.6 (1989): 569-585.

Nora, Lois Margaret., et al. "Improving Cross-cultural Skills of Medical Students Through Medical School-Community Partnerships." Western Journal of Medicine 161.2 (1994): 144-147.

Novello, Antonia Coello. "Hispanic Health: Time for data, time for action." Journal of the American Medical Association 265.2 (1991): 253-255.

-... "One Voice, One Vision--Uniting to Improve Hispanic-Latino Health." Public Health Reports 108.5 (1993): 529-533.

Omeri, Akram. Rev. of Transcultural Nursing: Concepts, Theories, Research, and Practices, by M. Leininger. Journal of Transcultural Nursing 7.2 (1996): 37-40. 
Oregon. Oregon Employment Department. 1990 Census Equal Employment Opportunity Detailed Report. Portland: 1990.

Oregon. Health Risk Factors in Oregon: County and Regional Estimates based on data from the Behavioral Risk Factor Surveillance System 1989-1994. Portland: Center for Health Statistics of the Oregon Health Division Oregon Department of Human Resources, 1996.

Oregon. Multnomah County Oregon Health Department Environmental Health Section: Childhood Lead Poisoning Prevention Program Environmental History Interview Form. 1996.

Oregon. Oregon State Board of Nursing: Annual Statistical Report Fiscal Year July 1, 1992-June 30, 1993. Portland: 1993.

Oregon. 68th Legislative Assembly Regular Session. "House Bill 2933" Salem: 1995.

Oregon Health Sciences University Summer Spanish Classes 1996.

Portland: Oregon Health Sciences University, 1996.

Oregon Health Sciences University Fall Spanish Classes 1996. Portland:

Oregon Health Sciences University, 1996.

Orque, Modesta Soberano., Bobbie Bloch and Lidia S. Ahumada Monrroy. Ethnic Nursing Care: A multicultural approach. St. Louis: C.V. Mosby, 1983.

Palacios, Carolina. "A Pilot Study Assessing the Health Status of the Hispanic American Community Living in Vancouver." Canadian 
Journal of Public Health 83.5 (1992): 46, 347-349.

Palerm, Juan Vicente. A season in the life of a migrant farm worker in California." The Western Journal of Medicine 157.3 (1992): 362366.

Panfilli, R. "Ethnocare of the Mexican-American." Diss. Wayne State U, 1983.

Phillips, Linda R., et al. "Focus on Psychometrics: Strategies for Achieving Cultural Equivalence." 17.2 (1994): 149-154.

Portland Community College Schedule: credit and non-credit classes Fall 1996. Portland: Portland Community College, 1996.

Putsch, Robert W. "Cross-Cultural Communication: The Special Case of Interpreters in Health Care." Journal of the American Medical Association 254.23 (1985): 3344-3348.

...., et al. "The medical interview across cultures." Patient Care 27.7 (1993): $141-144,146,148$.

-.-. "Dealing with Patients from Other Cultures." Clinical Methods: The History, Physical, and Laboratory Examinations. Boston: Butterworths, 1990. 1050-1065.

Rankin, Sandra B. "Developing Theraputic Relationships in Multicultural Settings." Academic Medicine 68.11 (1993): 826-827.

Reinert, Bonita R. "The Health Care Beliefs and Values of MexicanAmericans." Home Healthcare Nurse 4.5 (1986): 23, 26-27, 30- 
31.

Rivera, George. "Curanderismo and Childhood Illnesses." The Social Science Journal 23.3 (1986): 361-372.

Rojas, Dahlia. "Leadership in a multicultural society: a case in role development." Nursing \& Health Care 15.5 (1994): 258-261. Romano, Octavio Ignacio. "Charismatic Medicine, Folk-Healing and FolkSainthood." American Anthropologist 67.5 (1965): 1151-1173. Rooda, Linda. "Staff development for culturally sensitive nursing care." Journal of Nursing Staff Development 9.6 (1993): 262-265.

Root, Richard K. "Medical Staff Conference: Issues in Latino Health Care." The Western Journal of Medicine 146.2 (1987): 213-218.

Rosenbaum, Janet N. "A Cultural Assessment Guide: Learning cultural sensitivity." Canadian Nurse 87.4 (1991): 32-33.

Rubel, Arthur J. "Concepts of Disease in Mexican-American Culture." American Anthropologist 62.5 (1960): 795-814.

---. "The Epidemiology of a Folk Illness: Susto in Hispanic America." Ethnology 3.3 (1964): 268-283.

Ruiz, Pedro. "Access to Health Care for Uninsured Hispanics: Policy Recommendations." Hospital and Community Psychiatry 44.10 (1993): 958-962.

--. "Cultural Barriers to Effective Medical Care Among Hispanic-American Patients." Annual Reviews of Medicine 36.0 (1985): 63-71. 
Rust, George S. "Health Status of Migrant Farmworkers: A Literature Review and Commentary." American Journal of Public Health 80.10 (1990): $1213-1217$.

Ryan, Angela S. and Carmen Ortiz Hendricks. "Culture and Communication: Supervising the Asian and Hispanic Social Worker." The Clinical Supervisor 7.1 (1989): 27-40.

Samolsky, Susan., et al. "Feeding the Hispanic hospital patient: Cultural considerations." Journal of the American Dietetic Association 90.12 (1990): $1707-1710$.

Scheper-Hughes, Nancy. "Curanderismo in Taos County, New Mexico-A Possible Case of Anthropological Romanticism?" The Western Journal of Medicine 139.6 (1983): 875-884.

Scoville Baker, Susan and Natalie C. Burkhalter. "Teaching Transcultural Nursing in a Transcultural Setting." Journal of Transcultural Nursing 7.2 (1996): 10-13.

Scott, Clarissa S. "Health and Healing Practices Among Five Ethnic Groups in Miami, Florida." Public Health Reports 89.6 (1974): 524-532.

Shives, Louise Rebraca. Basic Concepts of Psychiatric-Mental Health Nursing. Philadelphia: J. B. Lippincott, 1986.

Siantz, M. "How can we become more aware of culturally specific body language and use this awareness theraputically?" Journal of 
Psychosocial Nursing \& Mental Health Services 29.11 (1991): 3841.

Simmons, Ozzie G. "Popular and Modern Medicine in Mestizo

Communities of Coastal Peru and Chile." Journal of American Folklore 68.267 (1955): 57-71.

Sincick, Gary. Personal interview. Oregon Employment Department. Aug. 1995.

Skolnick, Andrew A. "Clinic Explores Novel Approaches to Improving Health in Hispanic Community." Journal of the American Medical Association 273.19 (1995): 1475-1476.

Sluzki, Carlos E. "The Patient-Provider-Translator Triad:

A Note for Providers." Family Systems Medicine 2.4 (1984): $397-$ 400.

Smart, Julie F. "Cultural Issues in the rehabilitation of Hispanics." Journal of Rehabilitation 58.2 (1992): 29-37.

Smith, Peter J. "Lead Poisoning among Migrant Children In New York State." Public Health Briefs 66.4 (1976): 383-384.

Solis, Julia M. "Acculturation, Access to Care, and Use of Preventative Services by Hispanics: Findings from HHANES 1982-84." AJPH 80 Supplement (1990): 11-19.

Spector, Rachel E. Cultural Diversity in Health and Illness. Norwalk: Appleton \& Lange, 1991. 
Stedman, Thomas L. Stedman's Medical Dictionary. Baltimore: Williams \& Wilkins, 1982.

Susser, Mervyn. "Race, Health, and Health Services." American Journal of Public Health 83.7 (1993): 939. The National Coalition of Hispanic Health and Human Services Organizations (COSSMHO) Delivering Preventative Health Care To Hispanics: A Manual For Providers Washington D.C.: COSSMHO, 1990.

Thomas, Janice $\mathrm{T}$ and Lydia DeSantis. "Feeding and Weaning Practices of Cuban and Haitian Immigrant Mothers." Journal of Transcultural Nursing 6.2 (1995): $34-42$.

Thorne, Sally. "Health belief systems in perspective." Journal of Advanced Nursing 18.12 (1993): 1931-1941.

Thorngren, Mary. "Workshop A: The Effects of Ethnocultural Background on Access to and Use of Genetic Services; Health Care Concerns of Hispanic Populations. "Genetic Services for Underserved Populations 26.2 (1990): 39-45.

Thuman, Carol and Jane Maxwell. Where There /s No Doctor: a village health care handbook. Palo Alto: The Hesperian Foundation, 1992.

Trans-Interpreter: The Voice of Portland State University School of Extended Studies' Interpretation \& Translation Program. 2.1 (1996): 1-2. 
Treviño, Fernando M., et al. "Increasing the Representation of Hispanics in the Health Professions." Public Health Reports 108.5 (1993): $551-558$.

Tripp-Reimer, Toni. "Cultural Assessement: Content and Process." Nursing Outlook 32.2 (1984): 78-82.

---. "Retention of a Folk-Healing Practice (Matiasma) among Four Generations of Urban Greek Immigrants." Nursing Research 32.2 (1983): 97-101.

Trotter, Robert T. "Folk medicine in the Southwest." Folk Medicine 78.8 (1985): 167-179.

---. "Remedios Caseros: Mexican American Home Remedies and Community Health Problems." Social Science \& Medicine 15B (1981): $107-114$.

United States. Bureau of the Census. Statistical Abstract of the United States: 1994 114th ed. Washington DC: GPO, 1994.

United States. Department of Health and Human Services. Health United States 1994 Washington DC: GPO, 1994.

United States. National Center for Health Statistics. Health United States 1994 Hyattsville: Public Health Service, 1995.

Valdez, Burciaga R. "Improving Access to Health Care in Latino Communities." Public Health Reports 108.5 (1993): 534-539.

-.-. "Insuring Latinos Against the Costs of Illness." Journal of the 
American Medical Association 269.7 (1993): 889-894.

Waitzkin, Howard. "Doctor-Patient Communication: Clinical Implications of Social Scientific Research." Journal of the American Medical Association 252.17 (1984): 2441-2446.

Wallace, Steven and Chin-Yin Lew-Ting. "Cross-cultural Medicine A Decade Later: Getting By at Home Community-Based Long-term Care of Latino Elders." Western Journal of Medicine 157.3 (1992): 337-344.

Wanderer, Jules J. and George Rivera. "Black Magic Beliefs and White Magic Practices: The Common Structure of Intimacy, Tradition, and Power." The Social Science Journal 23.4 (1986): 419-430.

Warner, David C. "Health Issues at the US-Mexico Border." Journal of the American Medical Association 265.2 (1991): 242-247.

Weclew, Robert V. "The Nature, Prevalence, and Level of Awareness of 'Curanderismo' and Some of Its Implications for Community Mental Health." 11.2 (1975): 145-154.

Wells, Kenneth B. "Which Mexican-Americans Underutilize Health Services?" American Journal of Psychiatry 144.7 (1987): 918922.

West, Martin and Erin Moore. "Undocumented Workers in the United States and South Africa: A Comparative Study of Changing Control." Human Organization: Journal of the Society for Applied 
Anthropology 48.1 (1989): 1-10.

Williams, Ann B. "The Epidemiology, Clinical Manifestations and HealthMaintenance Need of Women Infected with HIV." 17.5 (1992): $27-38,41,44$.

Williams, Jane. "The Multicultural Workplace: Preparing Preceptors." The Journal of Continuing Education in Nursing 24.3 (1993): 101 104.

Woloshin, Steven. "Language Barriers in Medicine in the United States." The Journal of the American Medical Association 273.9 (1995): 724-728.

Wray, Linda A. "Cross-cultural Medicine A Decade Later: Health Policy and Ethnic Diversity in Older Americans." The Western Journal of Medicine 157.3 (1992): 357-361.

Wright, Barbara, and Nila Marrone. Language, Culture, and Health In Spanish-Speaking Societies: Spanish 190. Storrs: University of Connecticut, 1996.

Wright, Barbara. Spanish Language and Culture for Health Care Professions: Spanning Questions and Answers. Storrs: University of Connecticut, 1996.

Zambrana, Ruth E., et al. "The Relationship Between Psychological Status of Immigrant Latino Mothers and Use of Emergency Pediatric Services." Health \& Social Work 19.2 (1994): 93-102. 
Zuñiga, Maria E. "Using Metaphors in Therapy: Dichos and Latino Clients." Social Work 37.1 (1992): 55-60. 